Interfaces and Free Boundaries 12 (2010), 409-441

DOI 10.4171/IFB/240

\title{
Convergence of a large time-step scheme for mean curvature motion
}

\author{
E. CARLINI \\ Dipartimento di Matematica, Università Sapienza di Roma, \\ P.le Aldo Moro, 2, I-00185 Roma, Italy \\ E-mail: carlini@mat.uniroma1.it \\ M. FALCONE \\ Dipartimento di Matematica, Università Sapienza di Roma, \\ P.le Aldo Moro, 2, I-00185 Roma, Italy \\ E-mail:falcone@mat.uniromal.it \\ R. FERRETTI \\ Dipartimento di Matematica, Università di Roma Tre, \\ L.go S. Leonardo Murialdo, 1, I-00146 Roma, Italy \\ E-mail:ferretti@mat.uniroma3.it
}

[Received 18 July 2008 and in revised form 1 October 2010]

We analyse the properties of a semi-Lagrangian scheme for the approximation of the Mean Curvature Motion (MCM). This approximation is obtained by coupling a stochastic method for the approximation of characteristics (to be understood in a generalized sense) with a local interpolation. The main features of the scheme are that it can handle degeneracies, it is explicit and it allows for large time steps. We also propose a modified version of this scheme, for which monotonicity and consistency can be proved. Then convergence to the viscosity solution of the MCM equation follows by an extension of the Barles-Souganidis theorem. The scheme is also compared with similar existing schemes proposed by Crandall and Lions and, more recently, by Kohn and Serfaty. Finally, several numerical test problems in $2 \mathrm{D}$ and $3 \mathrm{D}$ are presented.

2010 Mathematics Subject Classification: Primary 65M12, 65M25; Secondary 49L25.

Keywords: Mean curvature motion; level-set approach; semi-Lagrangian schemes; consistency; generalized monotonicity; convergence.

\section{Introduction}

In this paper we analyse the properties of a semi-Lagrangian scheme for the approximation of the viscosity solutions $u(x, t)$ of the Mean Curvature Flow equation

$$
\begin{cases}u_{t}=\operatorname{trace}\left(\left(I-\frac{D u \otimes D u}{|D u|^{2}}\right) D^{2} u\right)=\operatorname{div}\left(\frac{D u}{|D u|}\right)|D u| & \text { in } \mathbb{R}^{n} \times(0, T), \\ u=u_{0} & \text { on } \mathbb{R}^{n} \times\{t=0\} .\end{cases}
$$

Here, $D u$ stands for the space gradient, $D^{2} u$ for the Hessian matrix of second space derivatives of $u$, and for any vector $p \in \mathbb{R}^{n}, p \otimes p=p p^{T}$. A first version of this scheme has been introduced in [18] where consistency was proved assuming that the gradient of the solution does not vanish, i.e., in the nonsingular case. In the present analysis we drop this assumption and introduce a new 
version of the scheme which can handle the singular case. In order to prove convergence, the main ingredient is a generalization of the result obtained for monotone schemes by Barles and Souganidis in [3]. Since a straightforward extension of the scheme in [18] is not monotone (even in this weaker setting), we add a vanishing artificial viscosity term, as proposed by Crandall and Lions in [9], and introduce a new discretization parameter to approximate characteristics. For this version of the scheme we prove consistency and monotonicity (in a generalized sense), and thus convergence.

To put this paper into perspective, let us recall that Crandall and Lions have developed in [9] an analysis of explicit finite difference schemes for a class of possibly degenerate parabolic equations of the form

$$
u_{t}-\operatorname{trace}\left(\Theta(x, D u) \Theta(x, D u)^{T} D^{2} u\right)=0 \quad \text { for } t>0, x \in \mathbb{R}^{n} .
$$

Here, $\Theta(x, p)$ is an $N \times M$ matrix valued function of $(x, p) \in \mathbb{R}^{n} \times \mathbb{R}^{n}$ and $A^{T}$ denotes the transpose of a matrix $A$. Note that the integer $M$ is arbitrary and the equation is degenerate if $\Theta(x, p)$ is not invertible.

Our particular case (1) corresponds to the choice

$$
\Theta(x, p)=\Theta(p)=I-\frac{p \otimes p}{|p|^{2}}
$$

(where $|\cdot|$ denotes the euclidean norm). It is well known that in our case $\Theta$ is discontinuous at $p=0$ and degenerate for $p \neq 0$. Since $\Theta(p)^{2}=\Theta(p)$ and $\Theta(p)=\Theta(p)^{T}, \Theta(p)$ is a projection matrix, which in fact projects the diffusion orthogonally with respect to the gradient. Thus, (1) is equivalent to 2 with $\Theta$ given by 3 .

We will work in the framework of level set methods using the theory of viscosity solutions, which allows one to deal with both singularities of solutions and degeneracies of the parabolic operator (see [1] and [8] for an introduction to this theory). Existence and uniqueness for the viscosity solutions to the Cauchy problem (1) have been proved independently by Evans and Spruck [15] and by Chen, Giga and Goto [7]. Starting from those pioneering papers, the last years have witnessed a great development of the theory of curvature related flows, as well as its application to various fields like phase transitions, image processing, fluid dynamics, material science and crystallography (see the books [29] and [27] for a review of interesting applications and simulations). We refer the interested reader to the lecture notes [21], [32] and [13] for the theory of viscosity solutions in this framework. But, despite the large number of analytical results concerning well-posedness and qualitative properties of viscosity solutions to this type of equations, the literature on numerical methods for the solution of (1) is considerably poorer. Following the first scheme proposed by Osher and Sethian in [28], new (and more efficient) methods, as well as some theoretical convergence result, have been developed. A general convergence theorem for numerical schemes for fully nonlinear second order equations was proved by Barles and Souganidis in [3], and has been applied to prove convergence of finite difference schemes in [9]. Convergence theory for the so called BMO algorithm [25] in discrete time and continuous space can be found in [12], [2]. For the literature on finite element approximations we refer the reader to [11] and [26]. A more complete idea of the related literature may be found in the books [29] and [27].

We note that the gap between theory and practice is due to a number of technical difficulties that make it hard to prove convergence and to determine a priori error bounds for numerical approximations. Some regularized problems have been introduced to circumvent the singularity 
at $p=0$, e.g., in [9] the following standard regularized problem has been proposed:

$$
u_{t}=\operatorname{trace}\left(\left(I-\frac{D u \otimes D u}{|D u|^{2}+\epsilon}\right) D^{2} u\right) .
$$

In [15], it has been proved that convergence of solutions $u_{\epsilon}$ of (4) as $\epsilon \rightarrow 0$ is locally uniform in $(t, x)$ as long as $u_{0} \in U C\left(\mathbb{R}^{n}\right)$ (where $U C\left(\mathbb{R}^{n}\right)$ denotes as usual the space of uniformly continuous functions on $\mathbb{R}^{n}$ ). This is also used in [9] for the proof of convergence, which relies on the abstract theorem by Barles and Souganidis (see Section 3 for more details). The paper [10] presents global error estimates on the convergence rate of this scheme (in the discrete sup norm), which clearly require a delicate coupling between $\Delta x, \Delta t$ and $\epsilon$. The search for a corresponding a priori error estimate for the scheme under consideration would definitely be an interesting issue, but it will not be addressed in this work.

The scheme presented in this paper will not make use of any regularized continuous problem. First, the method introduced in [18] is used as long as $|D u|$ is bounded away from zero: the stochastic dynamical system which is behind the degenerate parabolic operator is discretized and we obtain a system of "generalized characteristics" for the degenerate problem.

Second, in order to handle the singular case $|D u|=0$, the scheme proposed in [18] is modified by switching to an approximation of the heat equation (cf. 26) whenever $|D u|$ is below a given threshold (which will depend on $\Delta x$, for consistency reasons).

The stochastic problem behind this interpretation has been introduced and analysed by Buckdahn, Cardaliaguet and Quincampoix in [4] and Soner and Touzi in [30] and [31] (see also [20] for the relations between stochastic processes and viscosity solutions to second order problems). More recently, Kohn and Serfaty have given in [24] a discrete game interpretation, in which the degenerate parabolic operator is approximated by a time discretization of min-max type.

The semi-Lagrangian (SL in what follows) scheme under consideration allows for large time steps since the standard parabolic stability condition $\Delta t / \Delta x^{2} \leqslant C$, which is typical of explicit finite difference approximations, is not required. Actually, the proofs in this paper refer to the modified scheme where a new discretization parameter has been introduced and an artificial viscosity term has been added. Then a critical balance between all the discretization parameters is required to obtain at the same time consistency and monotonicity, although such a balance turns out anyway to be less restrictive than the one required in [9], even if we do not make use of the regularized problem. In practice, our scheme does not need such a strict tuning to work properly and we obtain an accurate numerical approximation under much broader conditions for the parameters (see Section 8).

To complete the scenario, we should mention that several results on the approximation of viscosity solutions via SL schemes can be found in [17] (see also the survey paper [33] for a general overview of SL schemes). The first experimental results on a SL approximation of the Mean Curvature flow problem were presented in [34].

The paper is organized as follows. In Section 2 we introduce the semi-Lagrangian scheme. In Section 3 we present a weaker version of the convergence result by Barles and Souganidis. In the following two sections, we prove the assumptions needed for the convergence result. In particular, in Section 4 we prove that the scheme is consistent, including the case in which the continuous gradient vanishes and/or the discrete gradient is "small". In Section 5 we present an analysis of monotonicity for a regularization based on the addition of a viscosity term, and in Section 6 a convergence result is proved for this regularized version of the scheme. In Section 7, we compare our scheme with the scheme proposed by Crandall and Lions in [9] and with the approach of Kohn and Serfaty in [24]. 
Finally, in Section 8 we present and comment some numerical tests in 2D and 3D. These tests include the evolution of a front with a saddle point which generates a nonempty interior (fattening), and the evolution of both a torus and a dumbbell with possible topology changes.

\section{Construction of the scheme}

In what follows, we will always assume that

$$
u_{0} \text { is continuous on } \mathbb{R}^{n} \text { and constant on } \mathbb{R}^{n} \cap\{|x|>S\}
$$

for some $S>0$. The following result holds true:

TheOREM 2.1 ([15]) Assume that $(5]$ holds. Then there exists a unique viscosity solution of (1) such that

$$
u \text { is constant on } \mathbb{R}^{n} \times[0, \infty) \cap\{|x|+t>R\}
$$

for some $R>0$, depending only on $S$.

The properties of the viscosity solution $u$ of our problem can be found in [15]. We just need to recall that $u \in C\left(\mathbb{R}^{n} \times[0, \infty)\right) \cap L^{\infty}\left(\mathbb{R}^{n} \times[0, \infty)\right)$ and (see [10])

$$
\|u\|_{W^{1, \infty}}<C .
$$

We will denote by $L_{u}$ the Lipschitz constant of $u$, so that

$$
|u(x, t)-u(y, t)| \leqslant L_{u}|x-y| \quad \text { for any } x, y \in \mathbb{R}^{n}, t \geqslant 0 .
$$

The viscosity solution of (1) satisfies a representation formula proved in [4, 30], which, for regular solutions and whenever $D u \neq 0$, can be written as

$$
u(x, t)=\mathbb{E}\left\{u_{0}(y(t ; x, t))\right\} \quad \text { for any }(x, t) \in \mathbb{R}^{n} \times(0, T) .
$$

$\mathbb{E}(\cdot)$ is the probabilistic expectation, $y(s ; x, t)$ is the position at time $s$ of the solution trajectory of the stochastic initial value problem originating at time $t$ from $x$; more precisely,

$$
\left\{\begin{array}{l}
d y(s ; x, t)=\sqrt{2} P(D u(y(s ; x, t), t-s)) d W(s) \\
y(0 ; x, t)=x
\end{array}\right.
$$

where $W$ is an $n$-dimensional standard Brownian motion and $P$ is an $n \times n$ matrix defined by

$$
P(p)=I-\frac{p p^{T}}{|p|^{2}} .
$$

It is important to note that the matrix $P$ in $(11)$ is a projection matrix, being symmetric (i.e. $P=P^{T}$ ) and idempotent (i.e. $P^{2}=P$ ). Writing the representation formula on the time interval $[t, t+\Delta t]$ gives

$$
u(x, t+\Delta t)=\mathbb{E}\{u(y(\Delta t ; x, t+\Delta t), t)\} .
$$

The semi-Lagrangian scheme parallels the representation formula written on a single time step (see [18] for details). In fact, 9] suggests that solutions of the stochastic differential equation (10) may 
be interpreted as characteristics in a generalized sense, this being the basic idea which is behind the construction of a SL scheme. We discretize $(10)$ according to the theory of weak convergence for numerical schemes for stochastic differential equations (SDE). The interested reader can find details of this theory in [23].

If $P$ is of rank $r$, then $P$ projects onto the space generated by the eigenvectors $v_{i}$ with eigenvalues $\lambda_{i}=1$, which generate the spectral decomposition (this space coincides with the space orthogonal to the vector $p$ ). The normalized eigenvectors, $v_{i}, i=1, \ldots, r$, are such that

$$
P=\sum_{i=1}^{r} v_{i} v_{i}^{T}
$$

Then there exists an $n \times(n-1)$ matrix $\sigma$ such that

$$
P=\sigma \sigma^{T} .
$$

Finding $\sigma$ is straightforward in the two-dimensional case. It is given by the only eigenvector $v_{1}^{T}$ with eigenvalue $\lambda_{1}=1$. We obtain

$$
P=\sigma \sigma^{T} \quad \text { with } \quad \sigma(D u)=\frac{1}{|D u|}\left(\begin{array}{c}
-u_{x_{2}} \\
u_{x_{1}}
\end{array}\right) .
$$

Since Section 8 will present some tests in dimension 3, we will also construct the matrix $\sigma$ in the three-dimensional case. From the spectral decomposition we find the eigenvectors:

$$
\begin{aligned}
& v_{1}^{T}=\left(\frac{-u_{x_{3}}}{\sqrt{u_{x_{1}}^{2}+u_{x_{3}}^{2}}}, 0, \frac{u_{x_{1}}}{\sqrt{u_{x_{1}}^{2}+u_{x_{3}}^{2}}}\right), \\
& v_{2}^{T}=\frac{1}{|D u|}\left(\frac{-u_{x_{1}} u_{x_{2}}}{\sqrt{u_{x_{1}}^{2}+u_{x_{3}}^{2}}}, \sqrt{u_{x_{1}}^{2}+u_{x_{3}}^{2}}, \frac{-u_{x_{2}} u_{x_{3}}}{\sqrt{u_{x_{1}}^{2}+u_{x_{3}}^{2}}}\right) .
\end{aligned}
$$

The new form of $\sigma$ will be written as $\sigma=\left(v_{1}, v_{2}\right)$ in the case $\sqrt{u_{x_{1}}^{2}+u_{x_{3}}^{2}} \neq 0$, and otherwise $\sigma=\left(d_{1}, d_{2}\right)$, where $d_{1}^{T}=(1,0,0)$ and $d_{2}^{T}=(0,0,1)$. Applying the decomposition 14 , we have

$$
P d W=\sigma \sigma^{T} d W=\sigma\left(\sigma^{T} d W\right)=\sigma d \widehat{W},
$$

with $d \widehat{W}$ the differential of a standard $(n-1)$-dimensional Brownian motion. Using (18), we can write $(10)$ as

$$
\left\{\begin{array}{l}
d y(s ; x, t)=\sqrt{2} \sigma(D u(y(s ; x, t), t-s)) d \widehat{W}(s), \\
y(0 ; x, t)=x .
\end{array}\right.
$$

We remark that the advantage of considering (19) instead of (10) is that the Brownian motion $\widehat{W}$ is one dimension lower than $W$.

In this paper, 19 will be discretized by a simple stochastic Euler scheme, although more accurate approximation schemes are available (cf. [23]). This is done to simplify the analysis of the scheme but the same approach can in principle be applied to higher order schemes (see, 
e.g., [19]). Of course, the application of such techniques to the MCM equation would require additional investigations. The stochastic Euler scheme reads

$$
\left\{\begin{array}{l}
y_{k+1}=y_{k}+\sqrt{2} \sigma\left(D u\left(y\left(t_{k} ; x, t\right), t-t_{k}\right)\right) \Delta \widehat{W}_{k}, \\
y_{0}=x
\end{array}\right.
$$

where $t_{k}=k \Delta t, k \in \mathbb{N}$, and $\Delta t$ is a constant time step. Even if $\Delta \widehat{W}_{k}$ should represent a Gaussian variable with mean 0 and variance $\Delta t$, to obtain first order convergence it suffices to have a variable with 2-point discrete probability density,

$$
P\left(\Delta \widehat{W}_{k}= \pm \sqrt{\Delta t}\right)=1 / 2
$$

for the one-dimensional Brownian, and with 4-point discrete probability density,

$$
P\left(\left(\Delta \widehat{W}_{k, 1}, \Delta \widehat{W}_{k, 2}\right)=( \pm \sqrt{\Delta t}, \pm \sqrt{\Delta t})\right)=1 / 4,
$$

for the two-dimensional Brownian.

For simplicity, we will write the full approximation scheme in $\mathbb{R}^{2}$.

Assumption (5) allows us to drop the analysis of boundary conditions. Setting up a space grid of step $\Delta x$, we will denote by $x_{j}$ the nodes of the lattice $\mathcal{G}_{\Delta x}$ where $j=\left(j_{1}, j_{2}\right)$ and $x_{j}=\left(x_{1, j}, x_{2, j}\right)$.

We proceed by introducing some notations. Given $w: \mathcal{G}_{\Delta x} \rightarrow \mathbb{R}$, we define the approximate gradient on $\mathcal{G}_{\Delta x}$ using centred finite differences

$$
D_{j}[w]=\frac{1}{2 \Delta x}\left(\begin{array}{l}
w\left(x_{1, j}+\Delta x, x_{2, j}\right)-w\left(x_{1, j}-\Delta x, x_{2, j}\right) \\
w\left(x_{1, j}, x_{2, j}+\Delta x\right)-w\left(x_{1, j}, x_{2, j}-\Delta x\right)
\end{array}\right) .
$$

The scheme (introduced in [18]) which results from the above discretization is now written at the node $x_{j}$ and at the $(n+1)$-th time step:

$$
u_{j}^{n+1}=\frac{1}{2}\left(I\left[u^{n}\right]\left(x_{j}+\sigma_{j}^{n} \sqrt{\Delta t}\right)+I\left[u^{n}\right]\left(x_{j}-\sigma_{j}^{n} \sqrt{\Delta t}\right)\right)
$$

where $u^{n}$ is identified with the vector of values at the nodes $u^{n}=\left(u_{j}^{n}\right)_{j \in \mathcal{G}_{\Delta x}}, I[w](x)$ is a generic numerical interpolation (in space) of a function or vector $w$, and $\sigma_{j}^{n}$ is defined by

$$
\sigma_{j}^{n}=\frac{\sqrt{2}}{\left|D_{j}\left[u^{n}\right]\right|}\left(\begin{array}{c}
D_{2, j}\left[u^{n}\right] \\
-D_{1, j}\left[u^{n}\right]
\end{array}\right) .
$$

We will possibly use the short notation $D_{j}^{n}$ for $D_{j}\left[u^{n}\right]$. We also denote by $\mathcal{D}(j)$ the set of indices of the nodes which appear in the approximation of $D_{j}^{n}$, i.e.,

$$
\mathcal{D}(j)=\left\{\left(j_{1}+1, j_{2}\right),\left(j_{1}-1, j_{2}\right),\left(j_{1}, j_{2}-1\right),\left(j_{1}, j_{2}+1\right)\right\} .
$$

In order to deal with the singular case, we now give a modified version of the scheme (24) for the Cauchy problem (1). This new definition reads

$$
u_{j}^{n+1} \equiv \begin{cases}\frac{1}{2}\left(I\left[u^{n}\right]\left(x_{j}+\sigma_{j}^{n} \sqrt{\Delta t}\right)+I\left[u^{n}\right]\left(x_{j}-\sigma_{j}^{n} \sqrt{\Delta t}\right)\right) & \text { if }\left|D_{j}^{n}\right|>C \Delta x^{s}, \\ \frac{1}{4} \sum_{i \in \mathcal{D}(j)} u_{i}^{n} & \text { if }\left|D_{j}^{n}\right| \leqslant C \Delta x^{s},\end{cases}
$$


and is complemented with the initial condition

$$
u_{j}^{0}=u_{0}\left(x_{j}\right)
$$

for any $j \in \mathbb{Z}^{2}$ and $n=0, \ldots, N$ with $N=[T / \Delta t]$. Here, $C$ and $s$ are positive parameters to be fixed, and $\sigma_{j}^{n}$ is defined by (25). This modification of the scheme for small gradients is suitable for the generalized consistency condition, as we will see in Section 4

We observe that the definition

$$
u_{j}^{n+1}=\frac{1}{4} \sum_{i \in \mathcal{D}(j)} u_{i}^{n}
$$

might be regarded as a centred difference approximation for the heat equation

$$
u_{t}=\varepsilon \Delta u \quad \text { in } \mathbb{R}^{2} \times(0, T)
$$

for

$$
\varepsilon=\frac{\Delta x^{2}}{4 \Delta t}
$$

as one can easily check by just writing the centred finite difference scheme and imposing the condition on $\varepsilon$ which makes the contributions of the terms on the diagonal disappear.

In what follows, we will write the scheme 26 in compact form as

$$
u_{j}^{n+1}=H\left(u^{n} ; j\right) .
$$

\section{A general convergence theorem}

The scheme 26 is quite simple to implement and gives accurate results also in very degenerate cases (see Section 8). Unfortunately, in this form it does not satisfy at the same time the standard requirements of consistency and monotonicity, regardless of the relationship between the discretization parameters. Hence, it does not fit the framework of Barles-Souganidis convergence theory [3] which, roughly speaking, states that any monotone, stable and consistent scheme converges to the exact solution provided there exists a comparison principle for the limiting equation.

Therefore, in order to develop a convergence theory for the scheme, we will first prove a weaker form of the Barles-Souganidis theorem, and then work on a modified version of the scheme, which can satisfy such a generalized setting - in particular, monotonicity will be assumed in a less restrictive form. In this section we will present the abstract convergence result, and give a sketch of its proof. For simplicity, the presentation will be given in dimension 2 .

The Cauchy problem under consideration is

$$
\begin{cases}u_{t}+F\left(D u, D^{2} u\right)=0 & \text { in } \mathbb{R}^{2} \times(0, T), \\ u=u_{0} & \text { on } \mathbb{R}^{2} \times\{t=0\} .\end{cases}
$$

The function $F: \mathbb{R}^{2} \times M^{2} \rightarrow \mathbb{R}$ (where $M^{2}$ is the space of $2 \times 2$ symmetric matrices) is a locally bounded Hamiltonian, $u: \mathbb{R}^{2} \times(0, T) \rightarrow \mathbb{R}$ denotes the continuous solution and $u_{0}: \mathbb{R}^{2} \rightarrow \mathbb{R}$ the initial condition $\left(u_{0} \in B U C\left(\mathbb{R}^{2}\right)\right.$, the set of bounded uniformly continuous functions). 
The Hamiltonian $F$ is assumed to be elliptic, i.e.

$$
F(p, A) \leqslant F(p, B) \quad \text { for all } p \in \mathbb{R}^{2} \text { and } A, B \in M^{2} \text { such that } A \geqslant B,
$$

where $A \geqslant B$ is the usual ordering of symmetric matrices $(A-B \geqslant 0)$.

It is known that if $F$ is uniformly continuous and $u_{0} \in B U C\left(\mathbb{R}^{2}\right)$, then 30 has a unique viscosity solution in $B U C\left(\mathbb{R}^{2} \times(0, T)\right.$ ) (see Ishii and Lions [22]).

We will assume that a comparison principle holds true for (30), i.e. if $u$ and $v$ are respectively a subsolution and a supersolution of $(30)$ on $\mathbb{R}^{2} \times(0, T)$, and $u(\cdot, 0) \leqslant v(\cdot, 0)$, then $u \leqslant v$.

Let us now consider a general scheme that is supposed to approximate [30]. Its abstract form on a lattice will be given by

$$
\begin{cases}u_{j}^{n+1}=S^{\Delta t}\left(u^{n} ; j\right) & \text { for } j \in \mathbb{Z}^{2}, n=0, \ldots, N-1, \\ u_{j}^{0}=u_{0}\left(x_{j}\right) & \text { for } j \in \mathbb{Z}^{2} .\end{cases}
$$

Note that we have highlighted $\Delta t$ as the discretization parameter, with the convention that the space discretization step $\Delta x$ should be chosen on the basis of the relationship $\Delta x=C \Delta t^{\gamma}$, where $C$ and $\gamma$ are positive constants.

As in the original theorem in [3], we require the following property of invariance with respect to the addition of constants:

$$
S^{\Delta t}(v+K ; j)=S^{\Delta t}(v ; j)+K \quad \text { for any } K \in \mathbb{R} \text { and } j \in \mathbb{Z}^{2},
$$

and the generalized condition for consistency is stated as follows.

DEFINITION 3.1 Let $\left(\Delta x_{m}, \Delta t_{m}\right)$ be generic sequences of discretization parameters, and let $\left(x_{j_{m}}, t_{n_{m}}\right) \in \mathcal{G}_{\Delta x_{m}} \times\left\{0, \ldots, \Delta t_{m} N\right\}$ be generic sequences of nodes such that, for $m \rightarrow \infty$,

$$
\left(\Delta x_{m}, \Delta t_{m}\right) \rightarrow 0 \quad \text { and } \quad\left(x_{j_{m}}, t_{n_{m}}\right) \rightarrow(x, t) .
$$

Let moreover $\phi \in C^{\infty}\left(\mathbb{R}^{2} \times(0, T]\right)$ and $\phi^{n_{m}-1} \equiv\left(\phi\left(x_{j_{m}}, t_{n_{m}-1}\right)\right)_{x_{j_{m}} \in \mathcal{G}_{\Delta x_{m}}}$. Then the scheme $S^{\Delta t}$ is said to be consistent if

$$
\begin{aligned}
\phi_{t}(x, t) & +\underline{F}\left(D \phi(x, t), D^{2} \phi(x, t)\right) \leqslant \liminf _{m \rightarrow \infty} \frac{\phi\left(x_{j_{m}}, t_{n_{m}}\right)-S^{\Delta t_{m}}\left(\phi^{n_{m}-1} ; j_{m}\right)}{\Delta t_{m}} \\
& \leqslant \limsup _{m \rightarrow \infty} \frac{\phi\left(x_{j_{m}}, t_{n_{m}}\right)-S^{\Delta t_{m}}\left(\phi^{n_{m}-1} ; j_{m}\right)}{\Delta t_{m}} \leqslant \phi_{t}(x, t)+\bar{F}\left(D \phi(x, t), D^{2} \phi(x, t)\right) .
\end{aligned}
$$

Here, the index of the sequence is $m, j_{m}$ and $n_{m}$ denoting the corresponding indices of a node with respect to the $m$-th space-time grid. Moreover, we define as usual

$$
\begin{aligned}
& \underline{F}\left(D \phi(x, t), D^{2} \phi(x, t)\right)=\liminf _{(y, s) \rightarrow(x, t)} F\left(D \phi(y, s), D^{2} \phi(y, s)\right), \\
& \bar{F}\left(D \phi(x, t), D^{2} \phi(x, t)\right)=\limsup _{(y, s) \rightarrow(x, t)} F\left(D \phi(y, s), D^{2} \phi(y, s)\right)
\end{aligned}
$$

( $F$ and $\bar{F}$ are respectively the lower and upper semicontinuous envelopes of $F$ ). Note that if $F$ is continuous, then in (35) the lim inf and lim sup must coincide, and the definition reduces to the usual definition of consistency.

The standard definition of monotonicity is also replaced by a generalized monotonicity assumption stated as follows. 
DEFINITION 3.2 Let $\left(\Delta x_{m}, \Delta t_{m}\right)$ and $\left(x_{j_{m}}, t_{n_{m}}\right)$ be generic sequences satisfying (34). Then the scheme $S^{\Delta t}$ is said to be monotone (in the generalized sense) if it satisfies the following conditions:

$$
\begin{array}{ll}
\text { if } \quad v_{j_{m}} \leqslant \phi_{j_{m}}^{n_{m}-1} & \text { then } \quad S^{\Delta t_{m}}\left(v ; j_{m}\right) \leqslant \widetilde{S}^{\Delta t_{m}}\left(\phi^{n_{m}-1} ; j_{m}\right)+o\left(\Delta t_{m}\right), \\
\text { if } \quad \phi_{j}^{n_{m}-1} \leqslant v_{j_{m}} & \text { then } \quad \tilde{S}^{\Delta t_{m}}\left(\phi^{n_{m}-1} ; j_{m}\right) \leqslant S^{\Delta t_{m}}\left(v ; j_{m}\right)+o\left(\Delta t_{m}\right),
\end{array}
$$

where $v: \mathcal{G}_{\Delta x_{m}} \rightarrow \mathbb{R}$ is a bounded set of node values, and $\widetilde{S}^{\Delta t}$ is a (possibly different) scheme consistent with (30) in $(x, t)$, in the sense that it satisfies (35) in $(x, t)$.

Now, consider $u^{n}=\left(u_{j}^{n}\right)_{j \in \mathbb{Z}^{2}}$ with $u_{j}^{n}$ the solution of 32) and its piecewise constant (in time) interpolation $u^{\Delta t}$ defined by

$$
u^{\Delta t}(x, t)= \begin{cases}I\left[u^{n}\right](x) & \text { if } t \in\left[t_{n}, t_{n+1}\right), \\ u_{0}(x) & \text { if } t \in[0, \Delta t)\end{cases}
$$

Here $I[\cdot]$ is assumed to be a general interpolation operator

$$
I\left[u^{n}\right](x)=\sum_{l \in \mathcal{I}(x)} \psi_{l}(x) u_{l}^{n}
$$

where $\left\{\psi_{l}\right\}$ is a basis of cardinal functions in $\mathbb{R}^{2}$ (which in particular satisfy the condition $\left.\sum_{l} \psi_{l}(x) \equiv 1\right)$, and $\mathcal{I}(x)$ is the set of indices involved in interpolating at the point $x$. We assume that the corresponding set of nodes is contained in a ball of radius $O(\Delta x)$ around $x$.

The interpolation operator also has to verify a relaxed monotonicity property:

$$
\text { if } \quad v_{j} \leqslant \eta_{j} \text { for any } j \in \mathcal{I}(x) \text { then } I[v](x) \leqslant I[\eta](x)+o(\Delta t)
$$

with $v \in B\left(\mathcal{G}_{\Delta x}\right)$ and $\eta=\left(\phi\left(x_{j}\right)\right)_{x_{j} \in \mathcal{G}_{\Delta x}}$, where $\phi(x)$ is a smooth function and $B(A)$ denotes the set of bounded functions defined on $A$. Moreover, $I[\cdot]$ satisfies

$$
|I[\eta](x)-\phi(x)|=o(\Delta t) \quad \text { for any } x \in \mathbb{R}^{2} .
$$

Note that, once $\Delta t$ and $\Delta x$ are related according to $\Delta x=C \Delta t^{\gamma}$, bounds (39)-(40) (which are usually written in terms of the space discretization parameter) may also be understood in terms of $\Delta t$. We will come back to this point at the end of Sec.5

We can now state an extended version of the convergence result given in [3]:

THEOREM 3.1 Assume (33), (35) and (36)-(40) hold. Let $u(x, t)$ be the unique viscosity solution of [30). Then $u^{\Delta t}(x, t) \rightarrow u(x, t)$ locally uniformly on $\mathbb{R}^{2} \times[0, T]$ as $\Delta t \rightarrow 0$.

Proof. Let $\bar{u}, \underline{u} \in B\left(\mathbb{R}^{2} \times[0, T]\right)$ be defined by

$$
\bar{u}(x, t)=\limsup _{\substack{(y, s) \rightarrow(x, t) \\ \Delta t \rightarrow 0}} u^{\Delta t}(y, s), \quad \underline{u}(x, t)=\liminf _{\substack{y, s) \rightarrow(x, t) \\ \Delta t \rightarrow 0}} u^{\Delta t}(y, s) .
$$

We claim that $\bar{u}(x, t), \underline{u}(x, t)$ are respectively a subsolution and a supersolution of 30 . Assume for the moment that the claim is true; then by the comparison principle $\bar{u}(x, t) \leqslant \underline{u}(x, t)$ on $\mathbb{R}^{2} \times(0, T]$. Since the opposite inequality is obvious by the definition of $\bar{u}(x, t)$ and $\underline{u}(x, t)$, we have

$$
u=\bar{u}=\underline{u}
$$


and $u$ is the unique continuous viscosity solution of (30). This fact together with (41) also implies the locally uniform convergence of $u^{\Delta t}$ to $u$.

Let us prove the previous claim. Let $(x, t)$ be a local maximum point of $\bar{u}-\phi$ on $\mathbb{R}^{2} \times(0, T]$ for some $\phi \in C^{\infty}\left(\mathbb{R}^{2} \times(0, T]\right)$. Without any loss of generality, we may assume that $(x, t)$ is a strict global maximum point for $\bar{u}-\phi$ and that $\bar{u}(x, t)=\phi(x, t)$. Then, by a standard result from viscosity theory, there exist two sequences $\Delta t_{m} \in \mathbb{R}^{+}$and $\left(y_{m}, \tau_{m}\right) \in \mathbb{R}^{2} \times[0, T]$, which are global maximum points for $u^{\Delta t_{m}}-\phi$, and as $m \rightarrow \infty$,

$$
\Delta t_{m} \rightarrow 0, \quad\left(y_{m}, \tau_{m}\right) \rightarrow(x, t), \quad u^{\Delta t_{m}}\left(y_{m}, \tau_{m}\right) \rightarrow \bar{u}(x, t) .
$$

Then for any $x$ and $t$ we have

$$
u^{\Delta t_{m}}(x, t) \leqslant \phi(x, t)+\xi_{m}
$$

with $\xi_{m}=\left(u^{\Delta t_{m}}-\phi\right)\left(y_{m}, \tau_{m}\right)$ (note that since $\bar{u}(x, t)=\phi(x, t)$, we have $\left.\xi_{m} \rightarrow 0\right)$.

Since, in general, $\left(y_{m}, \tau_{m}\right) \notin \mathcal{G}_{\Delta x_{m}}$, we need to reconstruct the value attained by $u^{\Delta t_{m}}$ at such points. By the definition of $u^{\Delta t}$, there exists a $t_{n_{m}}$ such that $\tau_{m} \in\left[t_{n_{m}}, t_{n_{m}+1}\right)$ and $u^{\Delta t_{m}}\left(y_{m}, \tau_{m}\right)=$ $u^{\Delta t_{m}}\left(y_{m}, t_{n_{m}}\right)$. Furthermore, by the definition of $I[\cdot]$ in 38 , there exists a set of indices $\mathcal{I}\left(y_{m}\right)$ such that

$$
I\left[u^{n_{m}}\right]\left(y_{m}\right)=\sum_{j \in \mathcal{I}\left(y_{m}\right)} \psi_{j}\left(y_{m}\right) u_{j}^{n_{m}} .
$$

Next, we apply (42) at $t=t_{n_{m}-1}, x=x_{j}$ with $j \in \mathcal{I}\left(y_{m}\right)$ and deduce, from (33) and the monotonicity property [36, that

$$
S^{\Delta t_{m}}\left(u^{n_{m}-1} ; j\right) \leqslant \tilde{S}^{\Delta t_{m}}\left(\phi^{n_{m}-1} ; j\right)+\xi_{m}+o\left(\Delta t_{m}\right) .
$$

By the definition of $u^{n_{m}}$, we have

$$
u_{j}^{n_{m}} \leqslant \widetilde{S}^{\Delta t_{m}}\left(\phi^{n_{m}-1} ; j\right)+\xi_{m}+o\left(\Delta t_{m}\right),
$$

which yields, by applying (38), 39],

$$
u^{\Delta t_{m}}\left(y_{m}, \tau_{m}\right) \leqslant \sum_{j \in \mathcal{I}\left(y_{m}\right)} \psi_{j}\left(y_{m}\right) \tilde{S}^{\Delta t_{m}}\left(\phi^{n_{m}-1} ; j\right)+\xi_{m}+o\left(\Delta t_{m}\right) .
$$

Now, recalling the definition of $\xi_{m}$, we get

$$
\phi\left(y_{m}, \tau_{m}\right) \leqslant \sum_{j \in \mathcal{I}\left(y_{m}\right)} \psi_{j}\left(y_{m}\right) \tilde{S}^{\Delta t_{m}}\left(\phi^{n_{m}-1} ; j\right)+o\left(\Delta t_{m}\right) .
$$

We claim now that $\phi\left(y_{m}, \tau_{m}\right)=\phi\left(y_{m}, t_{n_{m}}\right)+O\left(\Delta t_{m}^{2}\right)$. In fact, either $\tau_{m}=t_{n_{m}}$ (and the claim obviously holds), or $\tau_{m} \in\left(t_{n_{m}-1}, t_{n_{m}}\right)$. In the latter case, since $\left(u^{\Delta t_{m}}-\phi\right)\left(y_{m}, \cdot\right)$ has a maximum at $\tau_{m}$ and $u^{\Delta t_{m}}$ is constant in $\left(t_{n_{m}-1}, t_{n_{m}}\right)$, it follows that $\phi_{t}\left(y_{m}, \tau_{m}\right)=0$ and we have $\phi\left(y_{m}, \tau_{m}\right)=\phi\left(y_{m}, t_{n_{m}}\right)+O\left(\Delta t_{m}^{2}\right)$.

Using the previous claim in (44), we have

$$
\phi\left(y_{m}, t_{n_{m}}\right) \leqslant \sum_{j \in \mathcal{I}\left(y_{m}\right)} \psi_{j}\left(y_{m}\right) \tilde{S}^{\Delta t_{m}}\left(\phi^{n_{m}-1} ; j\right)+o\left(\Delta t_{m}\right)
$$


and, by (40),

$$
\phi\left(y_{m}, t_{n_{m}}\right)=I\left[\phi^{n_{m}}\right]\left(y_{m}\right)+o\left(\Delta t_{m}\right)=\sum_{j \in \mathcal{I}\left(y_{m}\right)} \psi_{j}\left(y_{m}\right) \phi\left(x_{j}, t_{n_{m}}\right)+o\left(\Delta t_{m}\right) .
$$

Now, 45, and 46, imply

$$
\liminf _{m \rightarrow \infty} \sum_{j \in \mathcal{I}\left(y_{m}\right)} \psi_{j}\left(y_{m}\right) \frac{\phi\left(x_{j}, t_{n_{m}}\right)-\widetilde{S}^{\Delta t_{m}}\left(\phi^{n_{m}-1} ; j\right)}{\Delta t_{m}}+o(1) \leqslant 0 .
$$

Finally, by the consistency property [35, we obtain the desired result:

$$
\phi_{t}(x, t)+\underline{F}\left(D \phi, D^{2} \phi\right)(x, t) \leqslant 0 .
$$

The proof that $\underline{u}$ is a supersolution follows the same arguments, with (36) replaced by (37). We leave this adaptation to the reader.

REMARK 3.2 Note that the consistency condition (35) might be reformulated so as to avoid any dependence on the variable $t$. In fact, adding and subtracting $\phi\left(x_{j}, t_{n-1}\right)$, we obtain

$$
\frac{\phi\left(x_{j}, t_{n}\right)-S^{\Delta t}\left(\phi^{n-1} ; j\right)}{\Delta t}=\frac{\phi\left(x_{j}, t_{n}\right)-\phi\left(x_{j}, t_{n-1}\right)}{\Delta t}+\frac{\phi\left(x_{j}, t_{n-1}\right)-S^{\Delta t}\left(\phi^{n-1} ; j\right)}{\Delta t} .
$$

On the right-hand side of (47), the first term necessarily converges to $\phi_{t}\left(x_{j}, t_{n}\right)$, so that (35) is equivalent to

$$
\begin{aligned}
\underline{F}\left(D \phi(x), D^{2} \phi(x)\right) & \leqslant \liminf _{m \rightarrow \infty} \frac{\phi\left(x_{j_{m}}\right)-S^{\Delta t_{m}}\left(\eta ; j_{m}\right)}{\Delta t_{m}} \\
& \leqslant \limsup _{m \rightarrow \infty} \frac{\phi\left(x_{j_{m}}\right)-S^{\Delta t_{m}}\left(\eta ; j_{m}\right)}{\Delta t_{m}} \leqslant \bar{F}\left(D \phi(x), D^{2} \phi(x)\right),
\end{aligned}
$$

for a function $\phi(x)$ depending on $x$ alone (with $\eta$ denoting the vector of node values of $\phi$ ), and $x_{j_{m}} \rightarrow x$.

REMARK 3.3 We conclude with a further remark, crucial for the application of this result to the scheme under consideration in this paper. When assuming that $(x, t)$ is a strict global maximum point for $u-\phi$, we can further suppose that the test function $\phi$ is globally Lipschitz continuous whenever the exact solution has this property. In fact, the argument used to pass from a local to a global extremum requires modifying the test function away from the point $(x, t)$. If the solution has a Lipschitz constant $L_{u}$, this can be accomplished by test functions with a somewhat higher, but still uniformly bounded, Lipschitz constant (e.g., $2 L_{u}$ ).

\section{Consistency}

As required by [35, the consistency of the scheme will be proved assuming that the function $\phi$ is smooth enough to allow for all the differentiations we need. Let $\eta$ be a vector containing the samples of $\phi$ at time $t_{n}$, so that $\eta_{j} \equiv \phi\left(x_{j}, t_{n}\right)$. We make the following assumptions on the interpolation error and on the accuracy of the gradient estimation:

$$
\begin{array}{ll}
\left\|I[\eta](\cdot)-\phi\left(\cdot, t_{n}\right)\right\|_{\infty} \leqslant C_{1} \Delta x^{r} & \text { for any } n \in \mathbb{N}, \\
\left|D_{j}[\eta]-D \phi\left(x_{j}, t_{n}\right)\right| \leqslant C_{2} \Delta x^{q} & \text { for any } n \in \mathbb{N}, x_{j} \in \mathcal{G}_{\Delta x},
\end{array}
$$


with $C_{1}$ and $C_{2}$ positive constants. Typically, (49)-(50) require that

$$
\left|D^{i} \phi(x, t)\right|<M, \quad i=1, \ldots, \max (r, q+1) .
$$

We also assume that

$$
\left|\phi_{t t}(x, t)\right|<M,
$$

in order to bound the truncation error for the Euler time approximation. In [51]-[52], $M$ is a generic positive constant.

Although this setting is general enough to allow for different options, our main focus in developing a convergence theory will be on two specific choices: on one hand, the centred difference estimation of the gradient, outlined in Section 2, which implies $q=2$; on the other hand, the choice of $I[\cdot]$ as a linear or bilinear interpolation (this would be required in order to achieve monotonicity, see Section 5, which results in $r=2$.

The analysis presented in the following subsections shows that the scheme 26) is consistent (in the generalized sense of (35) ) with the equation (11). At the level of notations, we will identify the hamiltonian function $F$ with the operator

$$
F\left(D \phi, D^{2} \phi\right)=-\operatorname{div}\left(\frac{D \phi}{|D \phi|}\right)|D \phi|=-\sigma(D \phi)^{T} D^{2}(\phi) \sigma(D \phi)
$$

since we will present the proofs in $\mathbb{R}^{2}$.

Note that, due to the switching between the two different definitions of the scheme, checking (35) may require mixing different techniques. If the gradient of the function at $(x, t)$ is bounded away from zero, then 35 reduces to the standard definition and a local truncation error may be computed, whereas if $D \phi(x, t)=0$, we need to apply condition 35 in its most general setting.

We will first express the function $H$ at a node $\left(x_{j}, t_{n}\right)$, respectively above and below the threshold. Next, this analysis will be applied to check condition 35.

\subsection{Discrete gradients above the threshold}

In this first subsection, we will assume that $\left|D_{j}[\eta]\right|>C \Delta x^{s}$. Since this condition itself does not ensure that $D \phi\left(x_{j}, t_{n}\right) \neq 0$, we have to consider this circumstance separately.

CASE 1: $D \phi\left(x_{j}, t_{n}\right) \neq 0$. In this first case, the consistency analysis may be carried out by essentially standard arguments. First, we need a bound on the Lipschitz constant of $\sigma(p)=$ $\frac{\sqrt{2}}{|p|}\left(\begin{array}{c}p_{2} \\ -p_{1}\end{array}\right)$ which will be useful in the following. The Jacobian matrix is

$$
J_{\sigma}(p)=\left(\begin{array}{cc}
-\frac{\sqrt{2} p_{1} p_{2}}{|p|^{2}|p|} & \frac{\sqrt{2}}{|p|}-\frac{\sqrt{2} p_{2}^{2}}{|p|^{2}|p|} \\
-\frac{\sqrt{2}}{|p|}+\frac{\sqrt{2} p_{1}^{2}}{|p|^{2}|p|} & \frac{\sqrt{2} p_{1} p_{2}}{|p|^{2}|p|}
\end{array}\right),
$$

with $\left|J_{\sigma}(p)\right|^{2}=4 /|p|^{2}$, computed in the Frobenius norm. On the other hand, from $\left|D_{j}[\eta]\right|>C \Delta x^{s}$ one has

$$
L_{\sigma_{j}^{n}}=\frac{2}{C \Delta x^{s}}
$$


(we note that it is possible, although less explicit, to compute this Lipschitz constant without differentiations). In order to get an error bound, we write the elementary block in the scheme 260 as

$$
\begin{aligned}
I[\eta]\left(x_{j}+\sigma\left(D_{j}[\eta]\right) \sqrt{\Delta t}\right)= & I[\eta]\left(x_{j}+\sigma\left(D_{j}[\eta]\right) \sqrt{\Delta t}\right)-\phi\left(x_{j}+\sigma\left(D_{j}[\eta]\right) \sqrt{\Delta t}, t_{n}\right) \\
& +\phi\left(x_{j}+\sigma\left(D_{j}[\eta]\right) \sqrt{\Delta t}, t_{n}\right)-\phi\left(x_{j}+\sigma\left(D \phi\left(x_{j}, t_{n}\right)\right) \sqrt{\Delta t}, t_{n}\right) \\
& +\phi\left(x_{j}+\sigma\left(D \phi\left(x_{j}, t_{n}\right)\right) \sqrt{\Delta t}, t_{n}\right) .
\end{aligned}
$$

We now estimate the right-hand side of [54]. Note that

$$
\left|I[\eta]\left(x_{j}+\sigma\left(D_{j}[\eta]\right) \sqrt{\Delta t}\right)-\phi\left(x_{j}+\sigma\left(D_{j}[\eta]\right) \sqrt{\Delta t}, t_{n}\right)\right| \leqslant C_{1} \Delta x^{r}
$$

and that

$$
\left|\phi\left(x_{j}+\sigma\left(D_{j}[\eta]\right) \sqrt{\Delta t}, t_{n}\right)-\phi\left(x_{j}+\sigma\left(D \phi\left(x_{j}, t_{n}\right)\right) \sqrt{\Delta t}, t_{n}\right)\right| \leqslant 2 \frac{C_{2} M}{C} \Delta x^{q-s} \sqrt{\Delta t} .
$$

Plugging (55) and (56) into (54) gives

$$
I[\eta]\left(x_{j}+\sigma\left(D_{j}[\eta]\right) \sqrt{\Delta t}\right)=\phi\left(x_{j}+\sigma\left(D \phi\left(x_{j}, t_{n}\right)\right) \sqrt{\Delta t}, t_{n}\right)+O\left(\Delta x^{r}\right)+O\left(\Delta x^{q-s} \sqrt{\Delta t}\right) .
$$

Using this expression (and the similar expression obtained for $I[\eta]\left(x_{j}-\sigma\left(D_{j}[\eta]\right) \sqrt{\Delta t}\right)$ ) into the scheme 26 , we get

$$
\begin{aligned}
H(\eta ; j)= & \frac{1}{2}\left(I[\eta]\left(x_{j}+\sigma\left(D_{j}[\eta]\right) \sqrt{\Delta t}\right)+I[\eta]\left(x_{j}-\sigma\left(D_{j}[\eta]\right) \sqrt{\Delta t}\right)\right) \\
= & \frac{1}{2}\left(\phi\left(x_{j}+\sigma\left(D \phi\left(x_{j}, t_{n}\right)\right) \sqrt{\Delta t}, t_{n}\right)+\phi\left(x_{j}-\sigma\left(D \phi\left(x_{j}, t_{n}\right)\right) \sqrt{\Delta t}, t_{n}\right)\right) \\
& +O\left(\Delta x^{r}\right)+O\left(\Delta x^{q-s} \sqrt{\Delta t}\right) .
\end{aligned}
$$

Expressing now the value at points $x_{j} \pm \sigma\left(D \phi\left(x_{j}, t_{n}\right)\right) \sqrt{\Delta t}$ by a third-order Taylor expansion, we obtain

$$
\begin{aligned}
& H(\eta ; j) \\
&=\frac{1}{2}\left(\phi\left(x_{j}, t_{n}\right)+\sqrt{\Delta t} \sigma\left(D \phi\left(x_{j}, t_{n}\right)\right) D \phi\left(x_{j}, t_{n}\right)+\Delta t \sigma\left(D \phi\left(x_{j}, t_{n}\right)\right)^{T} D^{2} \phi\left(x_{j}, t_{n}\right) \sigma\left(D \phi\left(x_{j}, t_{n}\right)\right)\right. \\
&\left.+T_{3}\left(\Delta t^{3 / 2} \sigma\left(D \phi\left(x_{j}, t_{n}\right)\right), D^{3} \phi\left(x_{j}, t_{n}\right)\right)+O\left(\Delta t^{2}\right)\right) \\
&+\frac{1}{2}\left(\phi\left(x_{j}, t_{n}\right)-\sqrt{\Delta t} \sigma\left(D \phi\left(x_{j}, t_{n}\right)\right) D \phi\left(x_{j}, t_{n}\right)+\Delta t \sigma\left(D \phi\left(x_{j}, t_{n}\right)\right)^{T} D^{2} \phi\left(x_{j}, t_{n}\right) \sigma\left(D \phi\left(x_{j}, t_{n}\right)\right)\right. \\
&\left.-T_{3}\left(\Delta t^{3 / 2} \sigma\left(D \phi\left(x_{j}, t_{n}\right)\right), D^{3} \phi\left(x_{j}, t_{n}\right)\right)+O\left(\Delta t^{2}\right)\right)+O\left(\Delta x^{r}\right)+O\left(\Delta x^{q-s} \sqrt{\Delta t}\right) \\
&= \phi\left(x_{j}, t_{n}\right)+\Delta t \operatorname{div}\left(\frac{D \phi\left(x_{j}, t_{n}\right)}{\left|D \phi\left(x_{j}, t_{n}\right)\right|}\right)\left|D \phi\left(x_{j}, t_{n}\right)\right|+O\left(\Delta x^{r}\right)+O\left(\Delta x^{q-s} \sqrt{\Delta t}\right)+O\left(\Delta t^{2}\right) .
\end{aligned}
$$

In the Taylor expansions we have not written the third term $T_{3}$ explicitly since all odd terms (so in particular the terms in $\Delta t^{1 / 2}$ and $\Delta t^{3 / 2}$ ) cancel due to the symmetry of the two points $x_{j} \pm$ $\sigma\left(D_{j}[\eta]\right) \sqrt{\Delta t}$. 
Note that since for a smooth solution $u$ (in particular, with $u_{t t}$ bounded),

$$
u\left(x_{j}, t_{n+1}\right)=u\left(x_{j}, t_{n}\right)+\Delta t \operatorname{div}\left(\frac{D u\left(x_{j}, t_{n}\right)}{\left|D u\left(x_{j}, t_{n}\right)\right|}\right)\left|D u\left(x_{j}, t_{n}\right)\right|+O\left(\Delta t^{2}\right),
$$

(58) would imply an explicit bound for the local truncation error of the scheme 26) in the form

$$
\mathcal{T}_{\Delta x, \Delta t}\left(x_{j}, t_{n}\right)=O\left(\frac{\Delta x^{r}}{\Delta t}\right)+O\left(\frac{\Delta x^{q-s}}{\Delta t^{1 / 2}}\right)+O(\Delta t),
$$

but this expression would only hold in the "large gradient" case. It should also be noted that in general we expect the points $x_{j} \pm \sigma\left(D_{j}[\eta]\right) \sqrt{\Delta t}$ to be "far" from $x_{j}$. In fact, the feet of the generalized characteristics starting at $x_{j}$ will belong to the neighbouring cells (i.e., the cells having $x_{j}$ as a common vertex) under the parabolic-type CFL condition

$$
\frac{\sqrt{\Delta t}}{\Delta x}|\sigma|_{\max } \leqslant 1 .
$$

However, when the reconstruction $I[\cdot]$ is piecewise linear or bilinear, we have $r=2$ so that the $\Delta t / \Delta x$ relationship [59] would result in a nonconsistent scheme.

CASE 2: $D \phi\left(x_{j}, t_{n}\right)=0$. In this second case, the computations above can be repeated, but we cannot state the convergence of $\sigma\left(D_{j}[\eta]\right)$ to $\sigma\left(D \phi\left(x_{j}, t_{n}\right)\right)$ since the latter is not defined. Therefore, any reference to $\sigma\left(D \phi\left(x_{j}, t_{n}\right)\right)$ should be avoided. In particular, we have

$$
I[\eta]\left(x_{j}+\sigma\left(D_{j}[\eta]\right) \sqrt{\Delta t}\right)=\phi\left(x_{j}+\sigma\left(D_{j}[\eta]\right) \sqrt{\Delta t}, t_{n}\right)+O\left(\Delta x^{r}\right) .
$$

Using this expression and the expression obtained for the symmetric point, we obtain

$$
H(\eta ; j)=\frac{1}{2}\left(\phi\left(x_{j}+\sigma\left(D_{j}[\eta]\right) \sqrt{\Delta t}, t_{n}\right)+\phi\left(x_{j}-\sigma\left(D_{j}[\eta]\right) \sqrt{\Delta t}, t_{n}\right)\right)+O\left(\Delta x^{r}\right) .
$$

Expressing the value at the points $x_{j} \pm \sigma\left(D_{j}[\eta]\right) \sqrt{\Delta t}$ by a third-order Taylor expansion, and cancelling symmetric odd terms, we finally replace $(57)$ by

$$
H(\eta ; j)=\phi\left(x_{j}, t_{n}\right)+\Delta t \sigma\left(D_{j}[\eta]\right)^{T} D^{2} \phi\left(x_{j}, t_{n}\right) \sigma\left(D_{j}[\eta]\right)+O\left(\Delta t^{2}\right)+O\left(\Delta x^{r}\right) .
$$

\subsection{Discrete gradients below the threshold}

In this subsection we assume that the discrete gradient satisfies the condition $\left|D_{j}[\eta]\right|<C \Delta x^{s}$.

Here, $H(\eta ; j)=\frac{1}{4} \sum_{i \in \mathcal{D}(j)} \eta_{i}$ and we have shown in Section 2 that the scheme amounts to a discretization of the Laplacian. We have therefore

$$
H(\eta ; j)=\phi\left(x_{j}, t_{n}\right)+\Delta t \varepsilon \Delta \phi\left(x_{j}, t_{n}\right)+O\left(\Delta t^{2}\right),
$$

with $\varepsilon=\Delta x^{2} /(4 \Delta t)$. Note that, in this framework, the scheme appears to be a discretization of first order with respect to $\Delta t$. In fact, this is not a limitation in treating the degenerate case. 


\subsection{Checking the generalized consistency condition}

In order to apply the definition of generalized consistency, we consider in the $x, t$ space a sequence of nodes $\left(x_{j_{m}}, t_{n_{m}}\right) \rightarrow(x, t)$. As in Section 3 the index $m$ refers to the space-time grid, and $\left(j_{m}, n_{m}\right)$ have the same meaning as in (34). Accordingly, $\eta$ contains the samples of a smooth function $\phi$ at time $t_{n_{m}}$.

We again split the proof according to the value of $D \phi(x, t)$.

CASE 1: $D \phi(x, t) \neq 0$. In this case, there exists a neighbourhood of $(x, t)$ on which the gradient $D \phi$ is bounded away from zero, so that, at least asymptotically, $\left|D \phi\left(x_{j_{m}}, t_{n_{m}}\right)\right| \geqslant C \Delta x^{s}$ and we can just work with the form of the scheme used above the threshold. Then by (57) we get, at $\left(x_{j_{m}}, t_{n_{m}}\right)$,

$$
\begin{aligned}
\frac{\left|\phi\left(x_{j_{m}}, t_{n_{m}+1}\right)-H\left(\eta ; j_{m}\right)\right|}{\Delta t}= & \phi_{t}\left(x_{j_{m}}, t_{n_{m}}\right)+F\left(D \phi\left(x_{j_{m}}, t_{n_{m}}\right), D^{2} \phi\left(x_{j_{m}}, t_{n_{m}}\right)\right) \\
& +O\left(\frac{\Delta x^{r}}{\Delta t}\right)+O\left(\frac{\Delta x^{q-s}}{\Delta t^{1 / 2}}\right)+O(\Delta t)
\end{aligned}
$$

which clearly holds also for $\left(x_{j_{m}}, t_{n_{m}}\right) \rightarrow(x, t)$, and satisfies 35 (in a classical form) provided $\Delta x=o\left(\Delta t^{1 / r}\right), \Delta x^{q-s}=o\left(\Delta t^{1 / 2}\right)$.

CASE 2: $D \phi(x, t)=0$. In this situation, sequences converging to $(x, t)$ may have subsequences of nodes both above and below the threshold. We single out two different cases.

CASE 2A: $D \phi(x, t)=0,\left|D \phi\left(x_{j_{m}}, t_{n_{m}}\right)\right| \geqslant C \Delta x^{s}$. By 60, we have

$$
\begin{aligned}
\frac{\phi\left(x_{j_{m}}, t_{n_{m}+1}\right)-H\left(\eta ; j_{m}\right)}{\Delta t}= & \frac{\phi\left(x_{j_{m}}, t_{n_{m}+1}\right)-\phi\left(x_{j_{m}}, t_{n_{m}}\right)}{\Delta t} \\
& -\sigma\left(D_{j_{m}}[\eta]\right)^{T} D^{2} \phi\left(x_{j_{m}}, t_{n_{m}}\right) \sigma\left(D_{j_{m}}[\eta]\right)+O(\Delta t)+O\left(\frac{\Delta x^{r}}{\Delta t}\right) .
\end{aligned}
$$

Note that, in the limit, the term $\sigma\left(D_{j_{m}}[\eta]\right)^{T} D^{2} \phi\left(x_{j_{m}}, t_{n_{m}}\right) \sigma\left(D_{j_{m}}[\eta]\right)$ may only be bounded by its liminf and lim sup so that we obtain

$$
\begin{aligned}
\underline{F}\left(D \phi\left(x_{j_{m}}, t_{n_{m}}\right), D^{2} \phi\left(x_{j_{m}}, t_{n_{m}}\right)\right)+o(1) & \leqslant-\sigma\left(D_{j_{m}}[\eta]\right)^{T} D^{2} \phi\left(x_{j_{m}}, t_{n_{m}}\right) \sigma\left(D_{j_{m}}[\eta]\right) \\
& \leqslant \bar{F}\left(D \phi\left(x_{j_{m}}, t_{n_{m}}\right), D^{2} \phi\left(x_{j_{m}}, t_{n_{m}}\right)\right)+o(1)
\end{aligned}
$$

and, under the condition $\Delta x^{r}=o(\Delta t), 35$ is satisfied.

CASE 2B: $D \phi(x, t)=0,\left|D \phi\left(x_{j_{m}}, t_{n_{m}}\right)\right|<C \Delta x^{s}$. In this case, 61) implies

$$
\frac{\phi\left(x_{j_{m}}, t_{n_{m}+1}\right)-H\left(\eta ; j_{m}\right)}{\Delta t}=\frac{\phi\left(x_{j_{m}}, t_{n_{m}+1}\right)-\phi\left(x_{j_{m}}, t_{n_{m}}\right)}{\Delta t}-\varepsilon \Delta \phi\left(x_{j_{m}}, t_{n_{m}}\right)+O(\Delta t) .
$$

Under the condition $\Delta x^{2}=o(\Delta t)$, we have $\epsilon \rightarrow 0$ for $\Delta t \rightarrow 0$, so that asymptotically

$$
\underline{F}\left(D \phi(x, t), D^{2} \phi(x, t)\right) \leqslant \varepsilon \Delta \phi\left(x_{j_{m}}, t_{n_{m}}\right) \leqslant \bar{F}\left(D \phi(x, t), D^{2} \phi(x, t)\right)
$$

and the generalized consistency condition is satisfied again. 
Conclusions. This section can be summarized in the following

Theorem 4.1 Assume (49)-(52) hold. Assume moreover that

$$
\Delta x^{\min (2, r)}=o(\Delta t) \quad \text { and } \quad \Delta x^{q-s}=o\left(\Delta t^{1 / 2}\right)
$$

for $\Delta t \rightarrow 0$. Then the scheme (26) satisfies the generalized consistency condition 35 .

\section{Monotonicity}

Since consistency has been proved in the previous section, and 33 is trivially satisfied, in order to apply the Barles-Souganidis theorem (in the version given in Section 33, generalized monotonicity should now be checked for the scheme under consideration. In general, we expect that any form of monotonicity of the scheme would require the reconstruction $I[\cdot]$ to be performed for a basis of positive functions; therefore in what follows we will assume that the reconstruction is bilinear, i.e. of the form

$$
I\left[u^{n}\right](x)=\sum_{j \in \mathcal{I}(x)} \psi_{j}(x) u_{j}^{n}=\sum_{j \in \mathcal{I}(x)} \psi_{j_{1}}\left(x_{1}\right) \psi_{j_{2}}\left(x_{2}\right) u_{j}^{n},
$$

where $j=\left(j_{1}, j_{2}\right)$ and $\psi_{j_{i}}(\cdot)$ is the one-dimensional $\mathbb{P}_{1}$ basis function centred at $j_{i} \Delta x$ (recall that this is a nonnegative function).

However, the scheme (26) (as stated) is not yet monotone, even with a bilinear reconstruction and in the generalized form of (36)-377. Therefore, we must modify the scheme to meet such assumptions. A first modification consists in introducing a new discretization step to approximate the stochastic trajectory. In particular, the scheme is modified when $\left|D_{j}^{n}\right|>C \Delta x^{s}$, by introducing a new parameter $\rho$. We have

$$
u_{j}^{n+1}=H_{\rho}\left(u^{n} ; j\right)
$$

where $H_{\rho}\left(u^{n} ; j\right)$ is defined as

$$
\begin{aligned}
& H_{\rho}\left(u^{n} ; j\right) \\
& \equiv \begin{cases}u_{j}^{n}+\Delta t \frac{1}{\rho^{2}}\left(\frac{1}{2} I\left[u^{n}\right]\left(x_{j}+\sigma_{j}^{n} \rho\right)+\frac{1}{2} I\left[u^{n}\right]\left(x_{j}-\sigma_{j}^{n} \rho\right)-u_{j}^{n}\right) & \text { if }\left|D_{j}^{n}\right|>C \Delta x^{s}, \\
\frac{1}{4} \sum_{i \in \mathcal{D}(j)} u_{i}^{n} & \text { if }\left|D_{j}^{n}\right| \leqslant C \Delta x^{s} .\end{cases}
\end{aligned}
$$

Following the ideas contained in [9], we will consider a scheme $\widehat{H}_{\rho}$ which results from the further introduction of a vanishing artificial viscosity in the scheme $H_{\rho}$. The motivation for such an adaptation is the following. The scheme (66) is monotone with respect to the nodes used only by the interpolation operator. On the other hand, the dependence on the values related to indices in $\mathcal{D}(j)$ is more complex, and the introduction of the artificial viscosity term allows one to regain a monotone relationship.

Therefore, we will prove the generalized monotonicity property for the modified scheme

$$
u_{j}^{n+1}=\widehat{H}_{\rho}\left(u^{n} ; j\right)
$$


where

$$
\widehat{H}_{\rho}\left(u^{n} ; j\right) \equiv \begin{cases}H_{\rho}\left(u^{n} ; j\right)+\Delta t \frac{W \Delta x}{\rho \Delta x^{s}} \frac{\sum_{i \in \mathcal{D}(j)} u_{i}^{n}-4 u_{j}^{n}}{\Delta x^{2}} & \text { if }\left|D_{j}^{n}\right|>C \Delta x^{s}, \\ \frac{1}{4} \sum_{i \in \mathcal{D}(j)} u_{i}^{n} & \text { if }\left|D_{j}^{n}\right| \leqslant C \Delta x^{s},\end{cases}
$$

with $W$ a positive constant. We make the standing assumption that

$$
\frac{\Delta x^{1-s}}{\rho} \rightarrow 0
$$

which together with 49, 50,51, , 64, ensures that $\widehat{H}_{\rho}(\eta ; j)$ is consistent with 30) (see Section 6.

We are now ready to check conditions $36-37$.

First inequality. To check [36, suppose now that for any sequence $\left(x_{j}, t_{n}\right) \equiv\left(x_{j_{m}}, t_{n_{m}}\right)$ satisfying [34, the following inequality holds:

$$
u_{j}^{n} \leqslant \eta_{j}^{n}
$$

where $u^{n} \in B\left(\mathcal{G}_{\Delta x}\right)$ is the solution of 66, and $\eta=\left(\phi\left(x_{j}, t_{n}\right)\right)_{x_{j} \in \mathcal{G}_{\Delta x}, n \in \mathbb{N}}$ with a set of test functions $\phi \in C^{\infty}\left(\mathbb{R}^{2} \times(0, T)\right)$, and uniformly Lipschitz continuous, with Lipschitz constant $L_{\phi}>L_{u}$ (see the remark after Theorem 3.1. Since the monotonicity property does not depend on the iteration $n$, with no loss of generality we can drop the dependence on $t$ of the test function $\phi$ (and consequently of $\eta$ ). Condition (36) can be recast in the form

$$
\widehat{H}_{\rho}\left(u^{n} ; j\right) \leqslant \widetilde{H}_{\rho}(\eta ; j)+o(\Delta t),
$$

where the choice of $\widetilde{H}_{\rho}$ may vary from one subcase to the other.

The proof discriminates between two main cases.

CASE 1: $D \phi(x) \neq 0$. In this case, for $\Delta x \rightarrow 0$, the condition $\left|D_{j}[\eta]\right|>C \Delta x^{s}$ is asymptotically satisfied.

We further consider two different possibilities:

CASE $1 \mathrm{~A}:\left|D_{j}^{n}\right|>C \Delta x^{s}$. Writing the definition of $\widehat{H}_{\rho}\left(u^{n}, j\right)$ as

$$
\widehat{H}_{\rho}\left(u^{n} ; j\right)=H_{\rho}\left(u^{n} ; j\right)+\frac{W \Delta t \Delta x^{1-s}}{\rho}\left(-\frac{4 u_{j}^{n}}{\Delta x^{2}}\right)+\frac{W \Delta t \Delta x^{1-s}}{\rho \Delta x^{2}} \sum_{i \in \mathcal{D}(j)} u_{i}^{n},
$$

we split the right-hand side into two parts and start by observing that, by the definitions of $H_{\rho}$ and $\eta$,

$$
\begin{aligned}
H_{\rho}\left(u^{n} ; j\right)+\frac{W \Delta t \Delta x^{1-s}}{\rho}\left(-\frac{4 u_{j}^{n}}{\Delta x^{2}}\right) \leqslant & \eta_{j}+\frac{\Delta t}{\rho^{2}}\left(\frac{1}{2} I[\eta]\left(x_{j}+\sigma_{j}^{n} \rho\right)+\frac{1}{2} I[\eta]\left(x_{j}-\sigma_{j}^{n} \rho\right)-\eta_{j}\right) \\
& +\frac{W \Delta t \Delta x^{1-s}}{\rho}\left(-\frac{4 \eta_{j}}{\Delta x^{2}}\right)
\end{aligned}
$$

where the inequality holds provided

$$
1-\frac{\Delta t}{\rho^{2}}-\frac{4 W \Delta t}{\rho \Delta x^{1+s}} \geqslant 0 .
$$


Adding and subtracting the same quantity, we can write (72) as

$$
\begin{aligned}
\widehat{H}_{\rho}\left(u^{n} ; j\right) \leqslant & \widehat{H}_{\rho}(\eta ; j)+\frac{\Delta t}{2 \rho^{2}}\left(I[\eta]\left(x_{j}+\rho \sigma_{j}^{n}\right)+I[\eta]\left(x_{j}-\rho \sigma_{j}^{n}\right)\right) \\
& -\frac{\Delta t}{2 \rho^{2}}\left(I[\eta]\left(x_{j}+\rho \sigma_{j}^{n}\right)+I[\eta]\left(x_{j}-\rho \sigma_{j}^{n}\right)\right)+o(\Delta t) .
\end{aligned}
$$

Then, by (74), we find that 76 holds if and only if

$$
\begin{aligned}
\frac{W \Delta t}{\rho \Delta x^{1+s}} \sum_{i \in \mathcal{D}(j)}\left(\eta_{i}-u_{i}^{n}\right) \geqslant & -\frac{\Delta t}{2 \rho^{2}}\left(I[\eta]\left(x_{j}+\rho \sigma\left(D_{j}[\eta]\right)\right)+I[\eta]\left(x_{j}-\rho \sigma\left(D_{j}[\eta]\right)\right)\right) \\
& +\frac{\Delta t}{2 \rho^{2}}\left(I[\eta]\left(x_{j}+\rho \sigma_{j}^{n}\right)+I[\eta]\left(x_{j}-\rho \sigma_{j}^{n}\right)\right)+o(\Delta t) .
\end{aligned}
$$

In order to prove (77), we observe that (53) holds and by assumption (64) we can bound by $o(\Delta t)$ the interpolation error for the (smooth) function $\phi$. Then the following estimate holds:

$$
\begin{aligned}
\frac{\Delta t}{2 \rho^{2}}\left|I[\eta]\left(x_{j} \pm \rho \sigma\left(D_{j}[\eta]\right)\right)-I[\eta]\left(x_{j} \pm \rho \sigma_{j}^{n}\right)\right| & \leqslant \frac{\Delta t}{2 \rho} L_{\phi}\left|\sigma\left(D_{j}[\eta]\right)-\sigma_{j}^{n}\right| \\
& \leqslant \frac{\Delta t}{2 \rho} \frac{L_{\phi} C}{\Delta x^{s}}\left|D_{j}[\eta]-D_{j}^{n}\right| \\
& \leqslant \frac{\Delta t}{2 \rho} \frac{\sqrt{2} L_{\phi} C}{\Delta x^{1+s}} \max _{i \in \mathcal{D}(j)}\left(\eta_{i}-u_{i}^{n}\right)
\end{aligned}
$$

where $L_{\phi}$ denotes the Lipschitz constant of the test function $\phi$ (which coincides with $\eta$ on the nodes). Now, if we assume that

$$
W>\sqrt{2} C L_{\phi},
$$

we get in turn

$$
\begin{aligned}
\frac{\Delta t W}{\rho \Delta x^{1+s}} \sum_{i \in \mathcal{D}(j)}\left(\eta_{i}-u_{i}^{n}\right) \geqslant & \frac{\Delta t \sqrt{2} C L_{\phi}}{\rho \Delta x^{1+s}} \max _{i \in \mathcal{D}(j)}\left(\eta_{i}-u_{i}^{n}\right) \\
\geqslant & -\frac{\Delta t}{2 \rho^{2}}\left(I[\eta]\left(x_{j}+\rho \sigma\left(D_{j}[\eta]\right)\right)-I[\eta]\left(x_{j}+\rho \sigma_{j}^{n}\right)\right. \\
& \left.+I[\eta]\left(x_{j}-\rho \sigma\left(D_{j}[\eta]\right)\right)-I[\eta]\left(x_{j}-\rho \sigma_{j}^{n}\right)\right) .
\end{aligned}
$$

This proves (77), and therefore (72).

We conclude that $\widehat{H}_{\rho}\left(u^{n} ; j\right)$ satisfies the first generalized monotonicity condition 36 if the assumptions of Theorem 4.1 are complemented with the following conditions:

$$
\left\{\begin{array}{l}
1-\frac{\Delta t}{\rho^{2}}-\frac{4 W \Delta t}{\rho \Delta x^{1+s}} \geqslant 0, \\
W \geqslant \sqrt{2} C L_{\phi} .
\end{array}\right.
$$

CASE 1в: $\left|D_{j}^{n}\right| \leqslant C \Delta x^{s}$. In this case, $D_{j}^{n}$ being a centred difference, we have

$$
u_{j+e_{i}}^{n}=u_{j-e_{i}}^{n}+O\left(\Delta x^{s+1}\right), \quad i=1,2,
$$


where $e_{i}$ is the canonical base in $\mathbb{R}^{2}$. Since $\phi \in C^{\infty}\left(\mathbb{R}^{2}\right)$, by a Taylor expansion we can write

$$
\phi(x)=\phi\left(x_{j}\right)+\left(x-x_{j}\right) D \phi\left(x_{j}\right)+\frac{1}{2}\left(x-x_{j}\right)^{T} D^{2} \phi\left(x_{j}\right)\left(x-x_{j}\right)+O\left(\left|x-x_{j}\right|^{3}\right) .
$$

By (71), we get

$$
u_{j \pm e_{i}}^{n} \leqslant \phi\left(x_{j} \pm e_{i} \Delta x\right)=\phi\left(x_{j}\right) \pm \Delta x e_{i} D \phi\left(x_{j}\right)+\frac{\Delta x^{2}}{2} e_{i}^{T} D^{2} \phi\left(x_{j}\right) e_{i}+O\left(\Delta x^{3}\right) .
$$

Our aim is to express the values $u_{j \pm e_{i}}^{n}$ using a negative first-order term from 80. To this end, we first consider the case in which $e_{i} D \phi\left(x_{j}\right)<0$. Then 80 may be rewritten as

$$
u_{j+e_{i}}^{n} \leqslant \phi\left(x_{j}+e_{i} \Delta x\right)=\phi\left(x_{j}\right)-\Delta x\left|e_{i} D \phi\left(x_{j}\right)\right|+\frac{\Delta x^{2}}{2} e_{i}^{T} D^{2} \phi\left(x_{j}\right) e_{i}+O\left(\Delta x^{3}\right),
$$

while, using (79), we also have

$$
u_{j-e_{i}}^{n}+O\left(\Delta x^{s+1}\right) \leqslant \phi\left(x_{j}\right)-\Delta x\left|e_{i} D \phi\left(x_{j}\right)\right|+\frac{\Delta x^{2}}{2} e_{i}^{T} D^{2} \phi\left(x_{j}\right) e_{i}+O\left(\Delta x^{3}\right),
$$

and therefore, plugging these estimates into 696 , we get

$$
\frac{1}{4} \sum_{i=1,2}\left(u_{j+e_{i}}^{n}+u_{j-e_{i}}^{n}\right) \leqslant \phi\left(x_{j}\right)-\frac{\Delta x}{2}\left(\left|e_{1} D \phi\left(x_{j}\right)\right|+\left|e_{2} D \phi\left(x_{j}\right)\right|\right)+\Delta x^{2} \Delta \phi\left(x_{j}\right)+O\left(\Delta x^{s+1}\right) .
$$

Note that if instead $e_{i} D \phi\left(x_{j}\right)>0$, 81) should be written for $u_{j-e_{i}}^{n}$ and 82] for $u_{j+e_{i}}^{n}$, the final result being equivalent.

Next, using the fact that $u^{n}$ satisfies (69), we add and subtract $\Delta t F\left(D \phi(x), D^{2} \phi(x)\right)$ (note that $\underline{F}=\bar{F}=F$, since we are in the case $D \phi(x) \neq 0$ ), obtaining

$$
\begin{aligned}
u_{j}^{n+1} \leqslant & -\frac{\Delta x}{2}\left(\left|e_{1} D \phi\left(x_{j}\right)\right|+\left|e_{2} D \phi\left(x_{j}\right)\right|\right)+\Delta x^{2} \Delta \phi\left(x_{j}\right)+\Delta t F\left(D \phi(x), D^{2} \phi(x)\right)+O\left(\Delta x^{s+1}\right) \\
& +\phi\left(x_{j}\right)-\Delta t F\left(D \phi(x), D^{2} \phi(x)\right) .
\end{aligned}
$$

Now, since $F\left(D \phi(x), D \phi^{2}(x)\right)$ is bounded for $\phi \in C^{\infty}$ and $\Delta t=o(\Delta x)$ from $(78)$, asymptotically for $\Delta x \rightarrow 0$ and $\Delta t \rightarrow 0$, the following inequality holds:

$$
-\frac{\Delta x}{2}\left|e_{1} D \phi\left(x_{j}\right)\right|+O\left(\Delta x^{s+1}\right)+\Delta x^{2} \Delta \phi\left(x_{j}\right)+\Delta t F\left(D \phi(x), D^{2} \phi(x)\right) \leqslant 0,
$$

and hence there exists a $\overline{\Delta t}$ such that for every $\Delta t<\overline{\Delta t}$,

$$
u_{j}^{n+1} \leqslant \phi\left(x_{j}\right)-\Delta t F\left(D \phi(x), D^{2} \phi(x)\right) .
$$

The scheme denoted by $\widetilde{H}_{\rho}(\eta ; j)$ in 72 may now be chosen as a generic scheme satisfying 35 at $x$. Since the test function $\phi$ does not depend on time, $\widetilde{H}_{\rho}(\eta ; j)$ satisfies

$$
\lim _{m \rightarrow \infty} \frac{\phi\left(x_{j_{m}}\right)-\widetilde{H}_{\rho_{m}}\left(\eta ; j_{m}\right)}{\Delta t_{m}}=F\left(D \phi(x), D^{2} \phi(x)\right),
$$


so that $\phi\left(x_{j}\right)-\widetilde{H}_{\rho}(\eta ; j)=\Delta t F\left(D \phi(x), D^{2} \phi(x)\right)+o(\Delta t)$ and we finally get

$$
u_{j}^{n+1} \leqslant \widetilde{H}_{\rho}(\eta ; j)+o(\Delta t) .
$$

CASE $2: D \phi(x)=0$. When $\left|D_{j}^{n}\right| \leqslant C \Delta x^{s}$ the scheme is clearly monotone (in the conventional sense) at the node $x_{j}$, being a convex combination of node values. For $\left|D_{j}^{n}\right| \geqslant C \Delta x^{s}$, the scheme still satisfies (36) since

$$
u_{j}^{n+1}=\widehat{H}_{\rho}\left(u^{n} ; j\right) \leqslant \widetilde{H}_{\rho}(\eta ; j)
$$

where $\widetilde{H}_{\rho}(\eta ; j)$ has been chosen in the form

$$
\begin{aligned}
\widetilde{H}_{\rho}(\eta ; j)= & \phi\left(x_{j}\right)+\frac{\Delta t}{\rho^{2}}\left(\frac{1}{2} I[\eta]\left(x_{j}+\sigma_{j}^{n} \rho\right)+\frac{1}{2} I[\eta]\left(x_{j}-\sigma_{j}^{n} \rho\right)-\phi\left(x_{j}\right)\right) \\
& +\Delta t \frac{W \Delta x}{\rho \Delta x^{s}} \frac{\sum_{i \in \mathcal{D}(j)} \phi\left(x_{i}\right)-4 \phi\left(x_{j}\right)}{\Delta x^{2}} .
\end{aligned}
$$

The inequality (84) holds because the upwind points $x_{j} \pm \sigma_{j}^{n} \rho$ are the same on the left and right term and the time step $\Delta t$ satisfies the first inequality in 78 . Moreover $\widetilde{H}_{\rho}$ is a consistent scheme, since under condition 70 ,

$$
\frac{\phi\left(x_{j}\right)-\widetilde{H}_{\rho}(\eta ; j)}{\Delta t}=\left(\sigma_{j}^{n}\right)^{T} D^{2} \phi\left(x_{j}\right) \sigma_{j}^{n}+o(\Delta t) .
$$

Therefore, following the same arguments used in Case 2a of the consistency proof,

$$
\begin{aligned}
\underline{F}\left(D \phi(x), D^{2} \phi(x)\right) & \leqslant \liminf _{m \rightarrow \infty} \frac{\phi\left(x_{j_{m}}\right)-\widetilde{H}_{\rho_{m}}\left(\eta ; j_{m}\right)}{\Delta t_{m}} \\
& \leqslant \limsup _{m \rightarrow \infty} \frac{\phi\left(x_{j_{m}}\right)-\widetilde{H}_{\rho_{m}}\left(\eta ; j_{m}\right)}{\Delta t_{m}} \leqslant \bar{F}\left(D \phi(x), D^{2} \phi(x)\right),
\end{aligned}
$$

and then 35 is satisfied by $\widetilde{H}_{\rho}$.

Second inequality. In the second step, we have to check assumption 37. We assume now that, for $\Delta t \rightarrow 0$ and $\left(x_{j}, t_{n}\right) \rightarrow(x, t)$,

$$
u_{j}^{n} \geqslant \eta_{j}
$$

We need to prove that the scheme $\widehat{H}_{\rho}$ satisfies (37), and more precisely

$$
\widehat{H}_{\rho}\left(u^{n} ; j\right) \geqslant \widetilde{H}_{\rho}(\eta ; j)+o(\Delta t),
$$

in which the choice of $\widetilde{H}_{\rho}$ will follow the same guidelines used in proving (36).

CASE 3: $D \phi(x) \neq 0$. As we have seen, for $\Delta x \rightarrow 0$, the condition $\left|D_{j}[\eta]\right|>C \Delta x^{s}$ is asymptotically satisfied.

We consider the same subcases as before: 
CASE 3A: $\left|D_{j}^{n}\right|>C \Delta x^{s}$. This case follows directly from the proof of Case 1a. In fact, provided 750 holds, in the same way we find that $\widehat{H}_{\rho}$ satisfies 86 if and only if

$$
\begin{aligned}
\frac{W \Delta t}{\rho \Delta x^{1+s}} \sum_{i \in \mathcal{D}(j)}\left(u_{i}^{n}-\eta_{i}\right) \geqslant & \frac{\Delta t}{2 \rho^{2}}\left(I[\eta]\left(x_{j}+\rho \sigma\left(D_{j}[\eta]\right)\right)+I[\eta]\left(x_{j}-\rho \sigma\left(D_{j}[\eta]\right)\right)\right) \\
& -\frac{\Delta t}{2 \rho^{2}}\left(I[\eta]\left(x_{j}+\rho \sigma_{j}^{n}\right)+I[\eta]\left(x_{j}-\rho \sigma_{j}^{n}\right)\right),
\end{aligned}
$$

and this is satisfied if (78) holds.

CASE $3 \mathrm{~B}:\left|D_{j}^{n}\right| \leqslant C \Delta x^{s}$. In this case, following the same arguments of Case $1 \mathrm{~b}$, we have, by 85,

$$
\frac{1}{4} \sum_{i=1,2}\left(u_{j+e_{i}}^{n}+u_{j-e_{i}}^{n}\right) \geqslant \phi\left(x_{j}\right)+\frac{\Delta x}{2}\left(\left|e_{1} D \phi\left(x_{j}\right)\right|+\left|e_{2} D \phi\left(x_{j}\right)\right|\right)+\Delta x^{2} \Delta \phi\left(x_{j}\right)+O\left(\Delta x^{s+1}\right) .
$$

Next, we add and subtract $\Delta t F\left(D \phi, D^{2} \phi\right)(x)$, obtaining

$$
\begin{aligned}
\widehat{H}_{\rho}\left(u^{n} ; j\right) \geqslant & \frac{\Delta x}{2}\left(\left|e_{1} D \phi\left(x_{j}\right)\right|+\left|e_{2} D \phi\left(x_{j}\right)\right|\right)+\Delta x^{2} \Delta \phi\left(x_{j}\right)+\Delta t F\left(D \phi(x), D^{2} \phi(x)\right)+O\left(\Delta x^{s+1}\right) \\
& +\phi\left(x_{j}\right)-\Delta t F\left(D \phi(x), D^{2} \phi(x)\right) .
\end{aligned}
$$

Now, since $F\left(D \phi(x), D \phi^{2}(x)\right)$ is bounded for $\phi \in C^{\infty}$, and $\Delta t=o(\Delta x)$ from (78), asymptotically for $\Delta x \rightarrow 0$ and $\Delta t \rightarrow 0$, the following inequality holds:

$$
\frac{\Delta x}{2}\left(\left|e_{1} D \phi\left(x_{j}\right)\right|+O\left(\Delta x^{s+1}\right)+\Delta x^{2} \Delta \phi\left(x_{j}\right)+\Delta t F\left(D \phi(x), D^{2} \phi(x)\right) \geqslant 0,\right.
$$

and hence there exists a $\overline{\Delta t}$ such that for every $\Delta t<\overline{\Delta t}$,

$$
\widehat{H}_{\rho}\left(u^{n} ; j\right) \geqslant \phi\left(x_{j}\right)-\Delta t F\left(D \phi(x), D^{2} \phi(x)\right) .
$$

As in Case $1 \mathrm{~b}$, we add and subtract a generic scheme $\widetilde{H}_{\rho}(\eta ; j)$ satisfying (35) in $x$, and we finally get

$$
\widehat{H}_{\rho}\left(u^{n} ; j\right) \geqslant \widetilde{H}_{\rho}(\eta ; j)+o(\Delta t) \text {. }
$$

CASE $4: D \phi(x)=0$. As already remarked, if $\left|D_{j}^{n}\right| \leqslant C \Delta x^{s}$ the scheme is monotone at $x_{j}$ by definition.

If $\left|D_{j}^{n}\right| \geqslant C \Delta x^{s}$, the scheme satisfies (37) since

$$
\widehat{H}_{\rho}\left(u^{n} ; j\right) \geqslant \widetilde{H}_{\rho}(\eta ; j)
$$

with $\widetilde{H}_{\rho}(\eta ; j)$ chosen as in Case 2 .

Conclusions. This section can be summarized in the following

Theorem 5.1 Let (49), 64), (70) and (78) hold true. Then the scheme 69) is monotone in the sense that it satisfies 36, 37]. 


\section{Convergence}

In order to establish convergence for the regularized scheme (69) we have to show that the scheme is consistent and monotone in the generalized sense.

In fact, in the case $\left|D_{j}^{n}\right|>C \Delta x^{s}$ the regularized scheme (69) is consistent with (1) only if both the diffusion term tends to zero (this is true if $70 \mathrm{p}$ holds) and the local truncation error for large gradients vanishes for vanishing $\Delta x, \Delta t$ and $\rho$. By arguments similar to the one used in Theorem 4.1, it is easy to see that

$$
\mathcal{T}_{\rho, \Delta x, \Delta t}\left(x_{j}, t_{n}\right)=O\left(\frac{\Delta x^{2}}{\rho^{2}}\right)+O\left(\frac{\Delta x^{2-s}}{\rho}\right)+O(\rho)+O(\Delta t)+O\left(\frac{\Delta x^{1-s}}{\rho}\right)
$$

(note that, of the two terms $O\left(\Delta x^{2-s} / \rho\right)$ and $O\left(\Delta x^{1-s} / \rho\right)$, only the latter is relevant). We can now apply Theorem 3.1, obtaining the following

THEOREM 6.1 If $(78)$ holds true and

$$
\mathcal{T}_{\rho, \Delta x, \Delta t}\left(x_{j}, t_{n}\right) \rightarrow 0
$$

for $(\rho, \Delta x, \Delta t) \rightarrow 0$, then the scheme 69$)$ is convergent to the unique viscosity solution of $(1)$.

It remains to show that conditions $(78),(70)$ and $(89)$ can actually be satisfied at the same time. Let us define $\Delta x=\rho^{\alpha}$ for $\alpha \in \mathbb{R}^{+}$. Then, examining the first two terms in the truncation error, we infer that $\alpha$ and $s$ should satisfy $\alpha>1$ and $0 \leqslant s \leqslant 1$.

It is interesting to tune the parameters in order to optimize the performances of the approximation scheme (69). From the first inequality in 788 , we obtain

$$
\Delta t=\frac{\rho \Delta x^{1+s}}{\frac{\Delta x^{1+s}}{\rho}+4 W}
$$

and since $\Delta x^{1+s} / \rho \rightarrow 0$, we can choose a $\Delta t$ such that $\Delta t \simeq \frac{\rho \Delta x^{1+s}}{4 W}$, for instance

$$
\Delta t=\rho^{1+\alpha(1+s)} .
$$

Finally, from the requirement (70), we have $\alpha>1 /(1-s)$. To sum up, we can choose $\Delta t, \Delta x$ as a function of $\rho$, with $\alpha$ and $s$ such that $0<s<1$, and

$$
\alpha>\frac{1}{1-s} .
$$

Note that even in this monotonic version of the scheme, the parabolic CFL condition 59 ) is violated. In fact, taking into account the parameter balance just obtained, we have

$$
\frac{\Delta x^{2}}{\Delta t}=\rho^{2 \alpha-1-\alpha(1+s)}=\rho^{\alpha(1-s)-1}
$$

so that this ratio vanishes as $\rho \rightarrow 0$.

In practice, the numerical tests have been carried out with the nonmonotonic version (26), which works properly under even weaker conditions (e.g., the relationship which optimizes the consistency rate (58)). 


\section{Comparison with existing schemes}

In this section we compare the scheme with existing methods which rely on similar techniques. In particular, we discuss in detail the relationship with the schemes proposed by Crandall-Lions (see [9]) and by Kohn-Serfaty (see [24]). We also note that the latter was first proposed in a slightly different form by Catté, Dibos and Koepfler in [6].

This comparison will focus on two main points: on one hand, the technique used to detect the correct direction for the diffusion, and on the other, the way to treat the singular case in which $D u=0$. To better compare the various approaches, the truncation errors of the schemes will also be considered.

\subsection{Comparison with the Crandall-Lions (CL) scheme}

First, we compare the SL scheme with the scheme proposed by Crandall and Lions in [9]. We treat the two-dimensional case for simplicity, although the same arguments still hold in any dimension.

The main difference between the two schemes consists in the treatment of both singularity and degeneracy of the MCM operator. More precisely, in the CL scheme such problems are avoided by replacing the matrix $\Theta$ (given by (3) in the equation of MCM) by the following one:

$$
\Theta_{\epsilon}(p)=I-\frac{p \otimes p}{|p|^{2}+\epsilon},
$$

with $\epsilon>0$. Denoting by $e_{j}(j=1,2)$ the canonical base of $\mathbb{R}^{2}$, we can write the CL scheme as

$$
u_{j}^{n+1}=H_{C L}\left(u^{n} ; j\right)
$$

where

$$
\begin{aligned}
H_{C L}\left(u^{n} ; j\right)= & u_{j}^{n}+\frac{\Delta t}{\rho^{2}}\left(I\left[u^{n}\right]\left(x_{j}+\rho \Theta_{\epsilon}\left(D_{j}\left[u^{n}\right]\right) e_{1}\right)+I\left[u^{n}\right]\left(x_{j}+\rho \Theta_{\epsilon}\left(D_{j}\left[u^{n}\right]\right) e_{2}\right)\right. \\
& \left.+I\left[u^{n}\right]\left(x_{j}-\rho \Theta_{\epsilon}\left(D_{j}\left[u^{n}\right]\right) e_{1}\right)+I\left[u^{n}\right]\left(x_{j}-\rho \Theta_{\epsilon}\left(D_{j}\left[u^{n}\right]\right) e_{2}\right)-u_{j}^{n}\right) .
\end{aligned}
$$

The scheme for which convergence is proved is

$$
u_{j}^{n+1}=H_{C L}\left(u^{n} ; j\right)+\frac{\Delta t K}{\rho \Delta x}\left(\sum_{i \in \mathcal{D}(j)} u_{i}^{n}-4 u_{j}^{n}\right) .
$$

It should be noted that the addition of $\epsilon$ makes the problem nonsingular and adds an isotropic viscosity. The comparison between the schemes $(90)$ and $(26)$ shows that, in the CL scheme, the choice of avoiding degeneracy and singularity by replacing $(3)$ with the full-rank matrix $\Theta_{\epsilon}$ results in using four points to discretize the second order term. Instead, the SL scheme exploits the degeneracy, as explained in Sec.2 2 so that only the two points $x_{j} \pm \rho \sigma_{j}^{n}$ are used and undesired isotropic diffusion is avoided as much as possible. Of course, the need to treat the singular case persists, and this leads to the branching in (26).

Another difference is in the tuning of the discretization parameters. In [9], it is required that

$$
\frac{\Delta x}{\rho^{2}} \rightarrow 0 \quad \text { as } \Delta x, \rho \rightarrow 0,
$$


whereas, as we have shown in the previous section, the SL scheme has to satisfy the weaker condition

$$
\frac{\Delta x}{\rho} \rightarrow 0 \quad \text { as } \Delta x, \rho \rightarrow 0
$$

(this is due to a sharper use of the estimate (55), with $r=2$ instead of $r=1$, as used for (90)). Convergence requires therefore a different balance of the parameters: while in the CL scheme the balance is

$$
\Delta t=O\left(\sqrt{\epsilon} \rho^{3} g(\rho)\right), \quad \Delta x=O\left(\rho^{2} g(\rho)\right)
$$

where $g(\rho) \rightarrow 0$ as $\rho \rightarrow 0$, in the SL scheme the parameters are tuned according to

$$
\Delta t=O\left(\rho^{1+\alpha(1+s)}\right), \quad \Delta x=O\left(\rho^{\alpha}\right)
$$

with $0<s<1$ and $\alpha>1 /(1-s)$.

Choosing $s<1 / 4$ we can set $\alpha=4 / 3$ and then we get

$$
\Delta t=O\left(\rho^{\left.1+\frac{4}{3}(1+s)\right)}\right), \quad \Delta x=O\left(\rho^{4 / 3}\right) .
$$

Since $1+\frac{4}{3}(1+s)<3$, the conclusion is that, given the discretization parameter $\rho$, the requirements on both $\Delta x$ and $\Delta t$ are more restrictive for (90) than for [26].

\subsection{Comparison with the Kohn-Serfaty (KS) scheme}

First, we note that, as proved in [30], we can write

$$
\operatorname{div}\left(\frac{D u(x, t)}{|D u(x, t)|}\right)|D u(x, t)|=\min _{a \in S^{1}, a \cdot D u=0}\left\{a^{T} D^{2} u(x, t) a\right\},
$$

and in turn, the right-hand side of 94 can be rewritten as

$$
\begin{aligned}
\min _{a \in S^{1}, a \cdot D u=0}\left\{a^{T} D^{2} u(x, t) a\right\} & =\min _{a \in S^{1}} \max \left\{a^{T} D^{2} u(x, t) a-a \cdot D u, a^{T} D^{2} u(x, t) a+a \cdot D u\right\} \\
& =a^{*}(x)^{T} D^{2} u(x, t) a^{*}(x)
\end{aligned}
$$

where we have denoted by $a^{*}(x)$ the minimizer (note that since $a \in S^{1}$, both $a^{*}$ and $-a^{*}$ are minimizers and the choice of the sign is irrelevant). Here and below, we keep the assumption 49 . on the interpolation error and make use of the fact that if two functions $f_{1}(a)$ and $f_{2}(a)$ depend continuously on $a$ and span the same set, their max is minimized when they attain the same value.

For the Kohn-Serfaty scheme, $u_{j}^{n+1}=H_{K S}\left(u^{n} ; j\right)$, where $H_{K S}(w ; j)$ is defined by

$$
H_{K S}(w ; j)=\min _{a \in S^{1}} \max \left\{I[w]\left(x_{j}+\sqrt{2 \Delta t} a\right), I[w]\left(x_{j}-\sqrt{2 \Delta t} a\right)\right\} .
$$

Note that in order to have computable quantities, the reconstruction $I[\cdot]$ has been introduced in the scheme (the original scheme was set up only in time-discrete form), and the consistency analysis is developed accordingly. Let now $w$ be again a vector containing the samples of a smooth solution at time $t_{n}$, so that $w_{j}=u\left(x_{j}, t_{n}\right)$. Denoting by $\bar{a}_{j}$ the minimizer in $(96)$, we have

$$
H_{K S}(w ; j)=I[w]\left(x_{j}+\sqrt{2 \Delta t} \bar{a}_{j}\right)
$$


The values within the max can be expressed as

$$
\begin{aligned}
I[w]\left(x_{j} \pm \sqrt{2 \Delta t} a\right)= & u\left(x_{j} \pm \sqrt{2 \Delta t} a, t_{n}\right)+O\left(\Delta x^{r}\right) \\
= & u\left(x_{j}, t_{n}\right) \pm \sqrt{2 \Delta t} D u\left(x_{j}, t_{n}\right) \cdot a+\Delta t a^{T} D^{2} u\left(x_{j}, t_{n}\right) a \\
& +O\left(\Delta t^{3 / 2}\right)+O\left(\Delta x^{r}\right) .
\end{aligned}
$$

The max of $I[w]\left(x_{j} \pm \sqrt{2 \Delta t} a\right)$ is minimized when the two values coincide, so that

$$
\begin{aligned}
u\left(x_{j}, t_{n}\right) & +\sqrt{2 \Delta t} D u\left(x_{j}, t_{n}\right) \cdot \bar{a}_{j}+\Delta t \bar{a}_{j}^{T} D^{2} u\left(x_{j}, t_{n}\right) \bar{a}_{j}+O\left(\Delta t^{3 / 2}\right)+O\left(\Delta x^{r}\right) \\
& =u\left(x_{j}, t_{n}\right)-\sqrt{2 \Delta t} D u\left(x_{j}, t_{n}\right) \cdot \bar{a}_{j}+\Delta t \bar{a}_{j}^{T} D^{2} u\left(x_{j}, t_{n}\right) \bar{a}_{j}+O\left(\Delta t^{3 / 2}\right)+O\left(\Delta x^{r}\right)
\end{aligned}
$$

and therefore

$$
\sqrt{2 \Delta t} D u\left(x_{j}, t_{n}\right) \cdot \bar{a}_{j}=O\left(\Delta t^{3 / 2}\right)+O\left(\Delta x^{r}\right) .
$$

Note that (100) shows that the min-max operation replaces the finite difference estimation in selecting the direction orthogonal to the gradient. However, the accuracy of this estimate is lower, and this affects the overall consistency rate, as shown by the truncation error:

$$
\mathcal{T}_{\Delta x, \Delta t}\left(x_{j}, t_{n}\right) \leqslant O\left(\Delta t^{1 / 2}\right)+O\left(\frac{\Delta x^{r}}{\Delta t}\right) .
$$

In turn, this choice has the definite advantage of giving a monotone scheme, at the price of a higher computational complexity (the finite difference computation of the approximate gradient is replaced by a minmax).

Moreover, the minmax formulation does not make use of the gradient, thus allowing one to avoid any special caution in treating the singular case.

\section{Numerical tests}

In this section we analyse the performances of the algorithm in terms of its accuracy and capability to treat singular cases, by means of some examples in two and three space dimensions. The scheme has been used in the version (26), so that in particular no artificial viscosity has been added. For the first test we can also compute numerical errors, since the exact solution is known.

TEST 1: Two-dimensional circle evolution. We consider the problem (1) with initial condition

$$
u_{0}(x)= \begin{cases}\frac{\left(R^{2}-|x|^{2}\right)^{4}}{R^{8}} & \text { if }|x|^{2}<R^{2} \\ 0 & \text { elsewhere }\end{cases}
$$

with $R=3$, whose exact solution, for $t<1 / 2$, is

$$
u(x, t)= \begin{cases}\frac{\left(R^{2}-2 t-|x|^{2}\right)^{4}}{R^{8}} & \text { if }|x|^{2}<R^{2}-2 t, \\ 0 & \text { elsewhere. }\end{cases}
$$


We recall that this classical benchmark describes the shrinking of a circle in $\mathbb{R}^{2}$. Tables 1 and 2 refer to the approximate solution computed at the final time $T=0.16$, on a domain given by the square $[-4,4]^{2}$. The errors are obtained by comparison with the exact solution on the grid nodes. We have used $\|\cdot\|_{\infty}$ and $\|\cdot\|_{1}$ discrete norms, which represent respectively

$$
\begin{gathered}
\left\|u(\cdot, T)-u_{.}^{N}\right\|_{\infty}=\frac{\max _{j \in \mathcal{G}_{\Delta x}}\left|u\left(x_{j}, T\right)-u_{j}^{N}\right|}{\max _{j \in \mathcal{G}_{\Delta x}}\left|u\left(x_{j}, T\right)\right|}, \\
\left\|u(\cdot, T)-u_{.}^{N}\right\|_{1}=\frac{\sum_{j \in \mathcal{G}_{\Delta x}}\left|u\left(x_{j}, T\right)-u_{j}^{N}\right|}{\sum_{j \in \mathcal{G}_{\Delta x}}\left|u\left(x_{j}, T\right)\right|} .
\end{gathered}
$$

We have chosen $\Delta t$ and the parameter $s$ according to the truncation errors evaluation and the constant $C$ in $(26)$ has been set at $C=0.1$. The estimate (58) suggests, as a possible balance scale between parameters, the relationship

$$
\Delta t=O(\Delta x), \quad s=1,
$$

so that the order of consistency would be $1 / 2$. Following such a rule, we have obtained in this test (see Table 1) a convergence rate that goes well beyond the theoretical value.

For a comparison, we also include (see Table 2) errors and CPU times for the min-max (KohnSerfaty) scheme. The minimum has been evaluated by a bisection method. The tolerance for the bisection method and the relation between the time and the space steps have been chosen such that the accuracies of the two schemes are comparable.

TABLE 1

Errors for Test 1 obtained by the SL scheme

\begin{tabular}{ccccccc}
\hline$\Delta x$ & $\Delta t$ & $\|\cdot\|_{\infty}$ & $\|\cdot\|_{1}$ & $L^{\infty}$-order & $L^{1}$-order & $\mathrm{CPU}$ \\
\hline 0.078 & 0.08 & $6.06 \cdot 10^{-2}$ & $9.07 \cdot 10^{-3}$ & & & $0.10 \mathrm{~s}$ \\
0.039 & 0.04 & $3.18 \cdot 10^{-2}$ & $4.40 \cdot 10^{-3}$ & 0.93 & 1.04 & $0.53 \mathrm{~s}$ \\
0.019 & 0.02 & $1.66 \cdot 10^{-2}$ & $2.07 \cdot 10^{-3}$ & 0.93 & 0.99 & $2.85 \mathrm{~s}$ \\
$9.8 \cdot 10^{-3}$ & 0.01 & $8.32 \cdot 10^{-3}$ & $1.03 \cdot 10^{-3}$ & 0.99 & 1.00 & $21.85 \mathrm{~s}$ \\
\hline
\end{tabular}

TABLE 2

Errors for Test 1 obtained by the min-max scheme

\begin{tabular}{ccccccc}
\hline$\Delta x$ & $\Delta t$ & $\|\cdot\|_{\infty}$ & $\|\cdot\|_{1}$ & $L^{\infty}$-order & $L^{1}$-order & $\mathrm{CPU}$ \\
\hline 0.078 & 0.16 & $6.30 \cdot 10^{-2}$ & $6.52 \cdot 10^{-3}$ & & & $0.67 \mathrm{~s}$ \\
0.039 & 0.08 & $3.80 \cdot 10^{-2}$ & $3.73 \cdot 10^{-3}$ & 0.72 & 0.80 & $7.09 \mathrm{~s}$ \\
0.019 & 0.04 & $2.03 \cdot 10^{-2}$ & $2.12 \cdot 10^{-3}$ & 0.90 & 0.81 & $57.5 \mathrm{~s}$ \\
$9.8 \cdot 10^{-3}$ & 0.02 & $7.80 \cdot 10^{-3}$ & $1.26 \cdot 10^{-3}$ & 1.37 & 0.75 & $8 \mathrm{~m} 4 \mathrm{~s}$ \\
\hline
\end{tabular}

TEST 2: Development of a nonempty interior. In the second test, we have used as initial condition the function

$$
u_{0}(x, y)=a^{4} x^{4}-a^{2} x^{2}+b^{2} y^{2}+1,
$$

which represents a saddle point at the origin (located at the critical level $u=1$ ). Figure 1 confirms a known feature of viscosity solutions of (1), predicted by the analysis in [14]. When a saddle point 

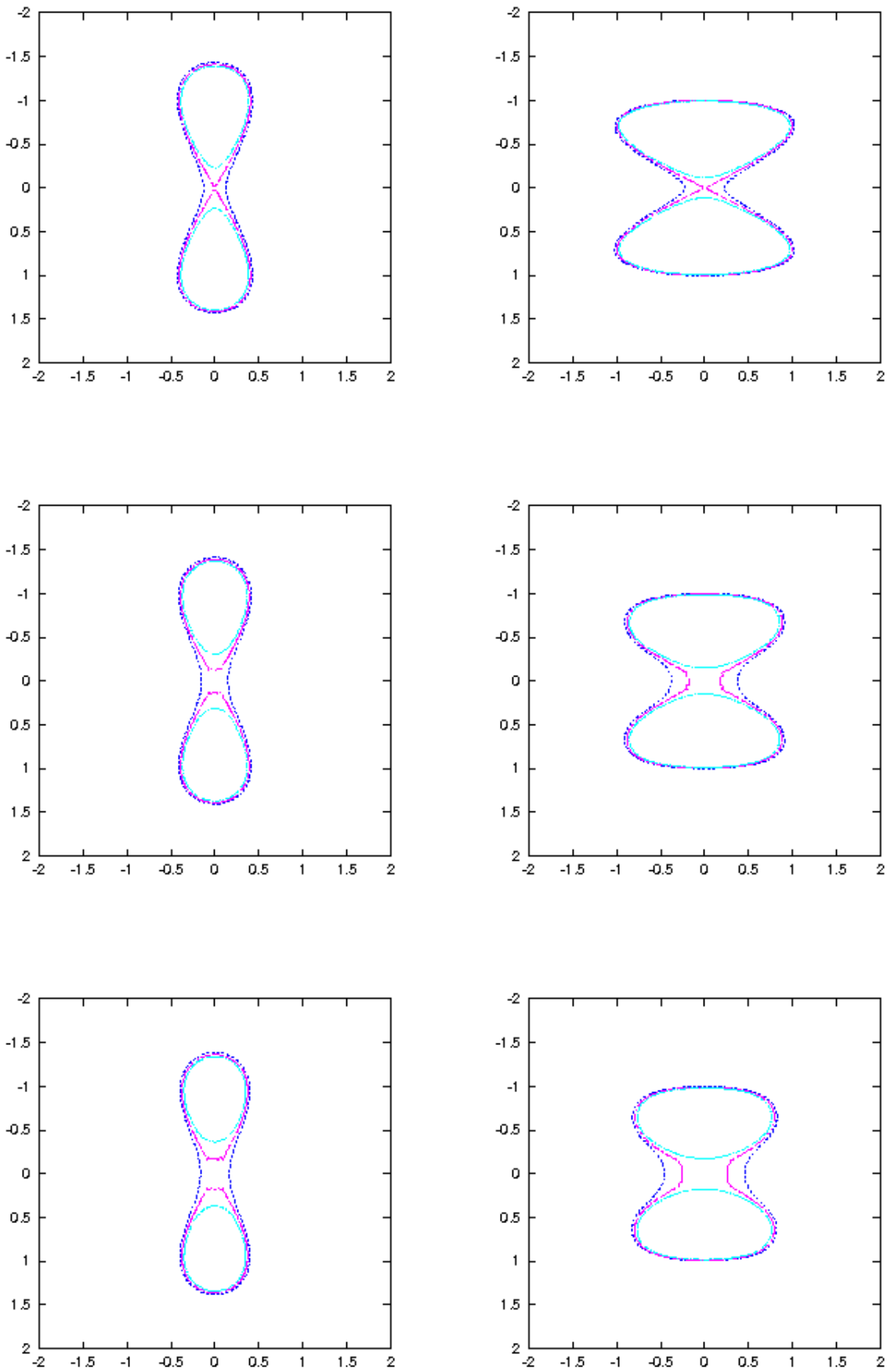

FIG. 1. Fattening: evolution of level curves around the critical value $u=1$. 

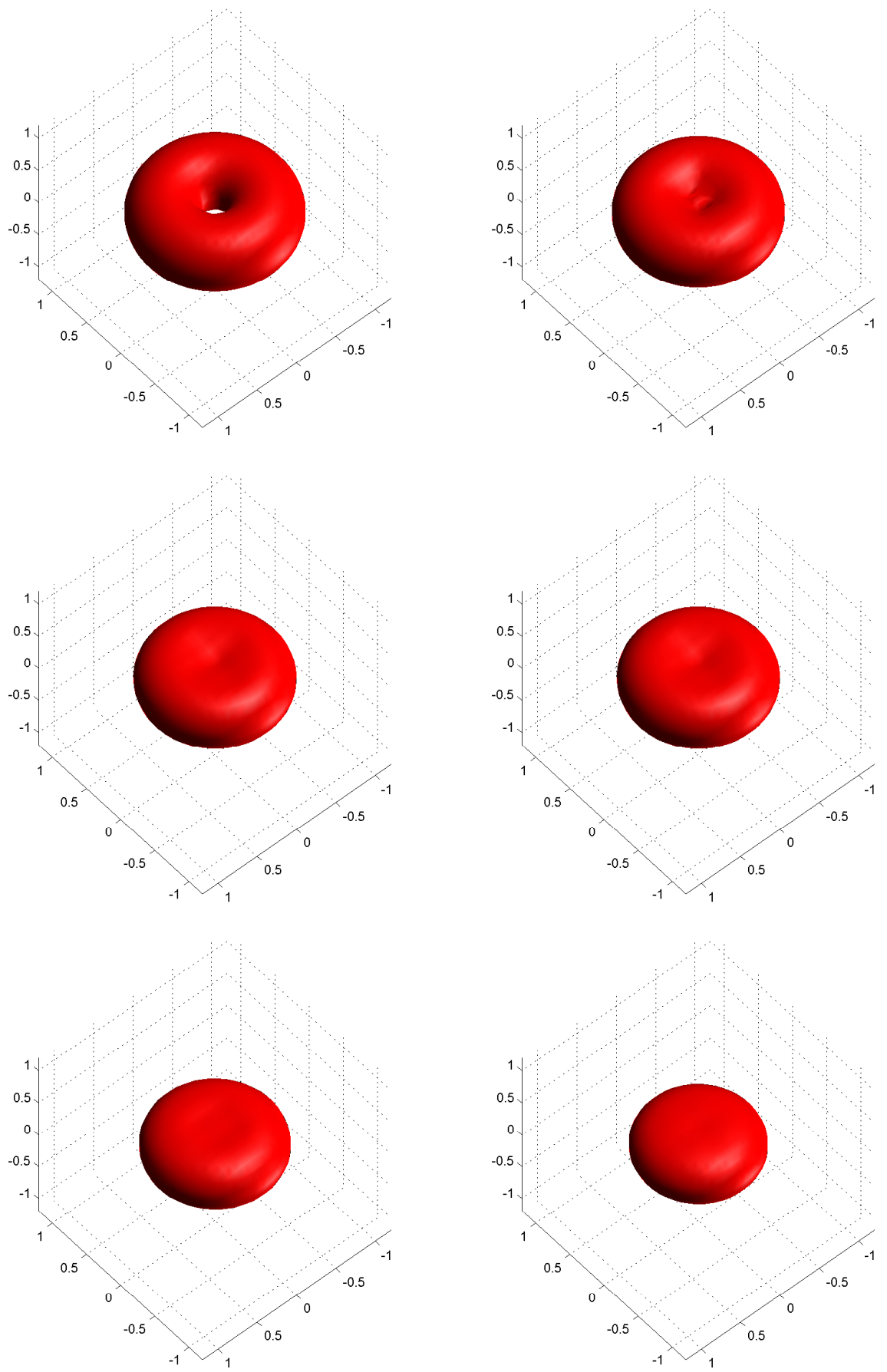

FIG. 2. The torus evolving into a sphere. 
MEAN CURVATURE MOTION
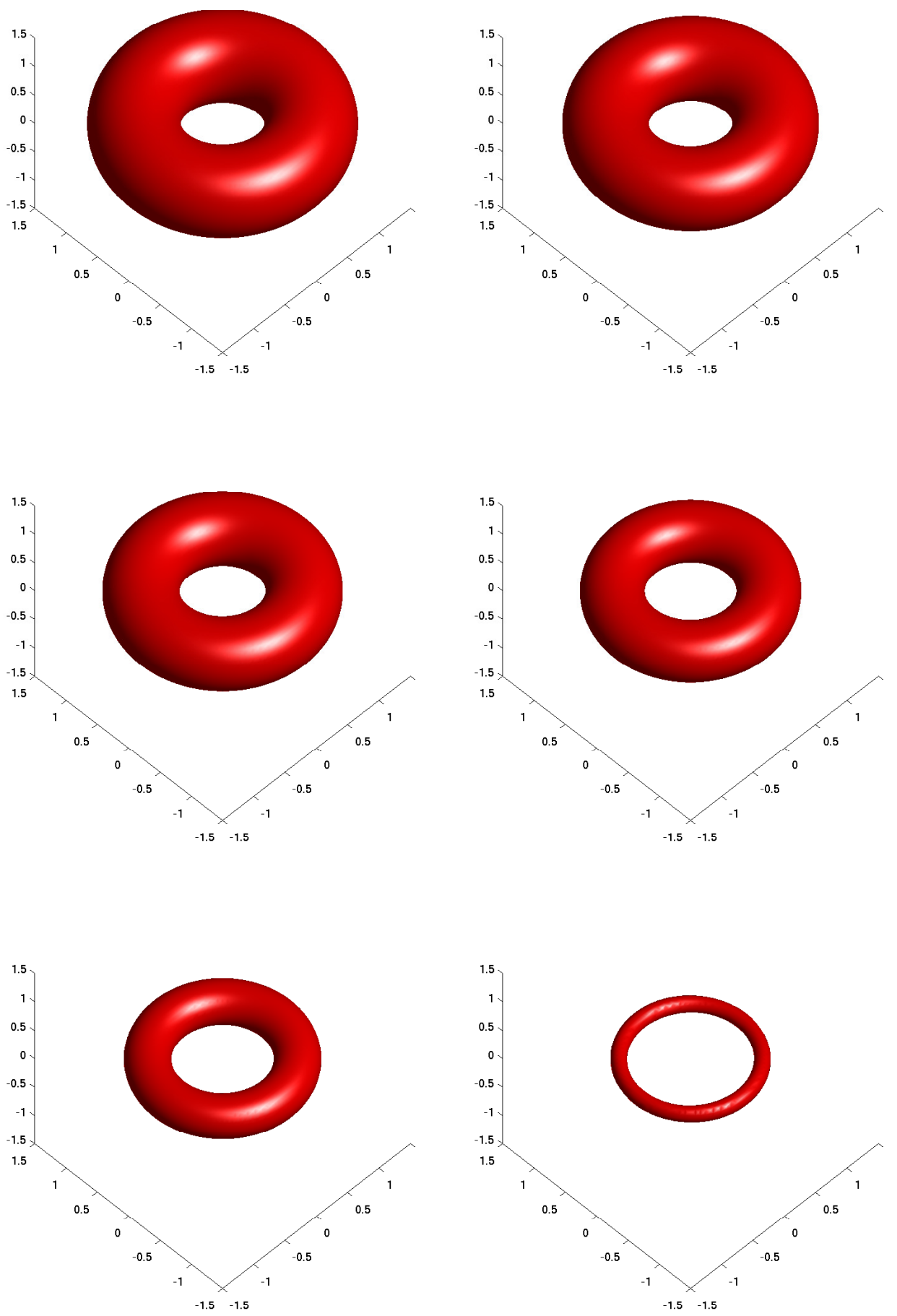

FIG. 3. The torus collapsing in a circle. 

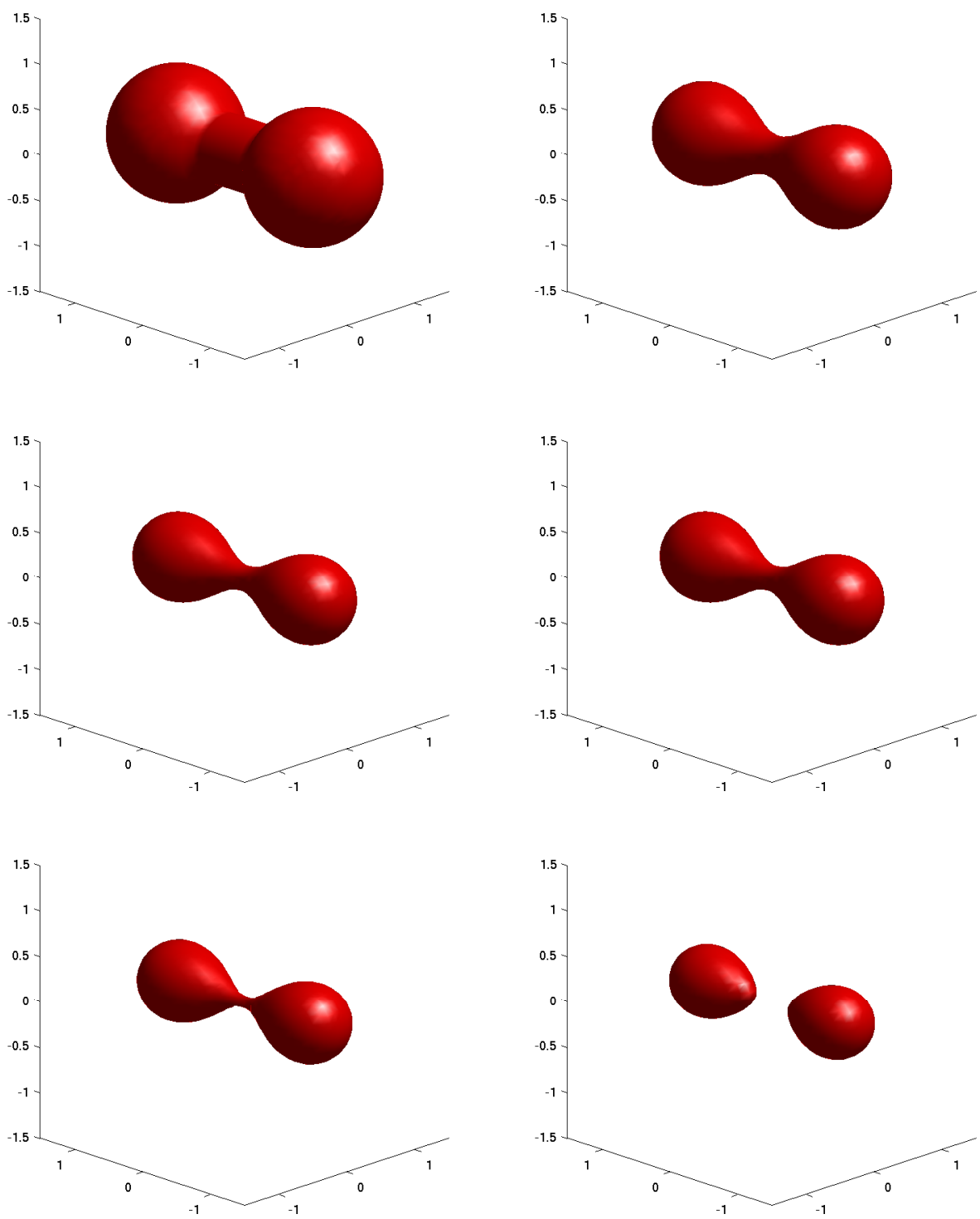

FIG. 4. Dumbbell: topology change in $\mathbb{R}^{3}$.

exists, the evolution associated to the critical level curve (which is not well-defined in the classical sense, since the level curve has a multiple point) develops an interior. At a numerical level, it is not reasonable to expect the existence of a region with a strictly constant value of the numerical solution, but the plot of the two level curves $u=1 \pm 0.05$ in Figure 1 shows that an almost constant region of the solution is propagating (we must say that in this example we have chosen $\Delta t=\Delta x^{3 / 2}$ in order to treat the very strong degeneracy). Moreover, changing the parameters $a$ and $b$ it is possible to interchange the roles of acute and obtuse angles at the double point, as shown in the two columns 
of Figure 1. Then the plots of the level curve $u=1$ show that the scheme tends to break the double point by keeping acute angles connected (this is supposed to be the physical behaviour).

TEST 3: Three-dimensional torus evolution. Let us consider the problem (1) in $[-2,2]^{3} \subset \mathbb{R}^{3}$ with initial condition

$$
u_{0}(x, y, z)=\left(\sqrt{x^{2}+y^{2}}-R^{2}\right)+z^{2}-r^{2} .
$$

This test describes the collapse of a torus in $\mathbb{R}^{3}$. Simulations are performed using a cubic interpolation with $\Delta x=0.04$; the time step is $\Delta t=0.01$ in the first test and $\Delta t=0.005$ in the second one.

The evolution of a torus may have two different behaviours depending on the ratio between the main and the secondary radius of the torus. This is shown in Figures 2 and 3 .

Figure 2 represents the evolution starting from a torus with main radius $R=0.5$ and secondary radius $r=0.3$. The pictures are plotted at time $t=0,0.01,0.02,0.03,0.04,0.05$. As expected for this case, the torus changes topology and collapses to a sphere.

Figure 3 represents the evolution starting from a torus with main radius $R=1$ and secondary radius $r=0.5$. The pictures are plotted at time $t=0,0.03,0.06,0.09,0.12,0.15$. In this case the different relationship between the main curvatures make the front collapse by shrinking to a circle, and without any topology change.

TEST 4: Three-dimensional dumbbell evolution. We lastly consider problem (1) in $[-2,2]^{3}$ with an initial condition describing a dumbbell. The test is performed using a cubic interpolation for $\Delta t=0.001$ and $\Delta x=0.08$.

Figure 4 shows the evolution of the surface corresponding to $t=0,0.04,0.06,0.08,0.09,0.1$. This is a typical situation in which a change in topology occurs. We see that the scheme follows the breaking of the dumbbell accurately and without spurious oscillations.

\section{REFERENCES}

1. BARles, G. Solutions de viscosité des équations de Hamilton-Jacobi. Math. Appl. 17, Springer, Paris (1994). Zbl 0819.35002 MR 1613876

2. Barles, G., \& Georgelin, CH. A simple proof of the convergence of an approximation scheme for computing Mean Curvature Motion. SIAM J. Numer. Anal. 32 (1995), 484-500. Zbl 0831.65138 MR 1324298

3. BARles, G., \& Souganidis, P. E. Convergence of approximation schemes for fully nonlinear secondorder equations. Asymptot. Anal. 4 (1991), 271-283. Zbl 0729.65077 MR 1115933

4. Buckdahn, R., Cardaliaguet, P., \& Quincampoix, M. A representation formula for the mean curvature motion. SIAM J. Math. Anal. 33 (2001), 827-846. Zbl 1074.93037| MR 1884724

5. Capuzzo Dolcetta, I., \& Lions, P.-L. (eds.) Viscosity Solutions and Applications (Montecatini Terme, 1995), Lecture Notes in Math. 1660, Springer, Berlin (1997). Zbl 0868.00050 MR 1462698

6. Catté, F., Dibos, F., \& Koepfler, G. A morphological scheme for mean curvature motion and applications to anisotropic diffusion and motion of level sets. SIAM J. Numer. Anal. 32 (1995), 1895-1909. Zbl 0841.68124 MR 1360464

7. Chen, Y. G., Giga, Y., \& Goto, S. Uniqueness and existence of viscosity solutions of generalized mean curvature flow equation. J. Differential Geom. 33 (1991), 749-786. Zbl 0696.35087| MR 1100211

8. Crandall, M. G., Ishit, H., \& LiONS, P.-L. User's guide to viscosity solutions of second order partial differential equations. Bull. Amer. Math. Soc. 27 (1992), 1-67. Zbl 0755.35015 MR 1118699 
9. Crandall, M. G., \& Lions, P.-L. Convergent difference schemes for nonlinear parabolic equations and mean curvature motion. Numer. Math. 75 (1996), 17-41. Zbl 0874.65066 MR 1417861

10. DeCKelnick, K. Error bounds for a difference scheme approximating viscosity solutions of mean curvature flow. Interfaces Free Bound. 2 (2000), 117-142. Zbl 0997.65112 2 MR 1760409

11. Dziuk, G., \& DeCKelnick, K. Convergence of numerical schemes for the approximation of level set solutions to mean curvature flow. In [16], pp. 77-94. Zbl 0989.65103 MR 1886708

12. Evans, L. C. Convergence of an algorithm for mean curvature motion. Indiana Univ. Math. J. 42 (1993), 533-557. Zbl 0802.65098 MR 1237058

13. Evans, L. C. Regularity for fully nonlinear elliptic equations and motion by mean curvature. In [5], pp. 98-133. Zbl 0905.35021 MR 1462701

14. Evans, L. C., Soner, H. M., \& Souganidis, P. E. Phase transitions and generalized motion by mean curvature. Comm. Pure Appl. Math. 45 (1992), 1097-1123. Zbl 0801.35045 MR 1177477

15. Evans, L. C., \& Spruck, J. Motion of level sets by mean curvature I. J. Differential Geom. 33 (1991), 635-681. Zbl 0726.53029 MR 1100206

16. FAlCONE, M., \& MAKRIDAKIS, CH. (eds.) Numerical Methods for Viscosity Solutions and Applications. World Sci. (2001). Zbl 0973.00022

17. Falcone, M., \& Ferretti, R. Semi-Lagrangian schemes for Hamilton-Jacobi equations, discrete representation formulae and Godunov methods. J. Comput. Phys. 175 (2002), 559-575. Zbl 1007.65060 MR 1880118

18. Falcone, M., \& Ferretti, R. Consistency of a large time-step scheme for mean curvature motion. In: Numerical Mathematics and Advanced Applications-ENUMATH 2001, F. Brezzi et al. (eds.), Springer (2003), 495-512. Zbl 1045.65072 MR 2360748

19. Ferretti, R. A technique for high-order treatment of diffusion terms in semi-lagrangian schemes. Comm. Comput. Phys., to appear.

20. Fleming, W. H., \& Soner, H. M. Controlled Markov Processes and Viscosity Solutions. Springer, New York (1993). Zbl 0773.60070 MR 1199811

21. GigA, Y. Surface evolution equations-a level set method. Hokkaido Univ. Technical Report Series in Mathematics, no. 71 (2002).

22. IsHiI, H., \& LiONS, P.-L. Viscosity solutions of fully nonlinear second order elliptic partial differential equations. J. Differential Equations 83 (1990), 26-78. Zbl 0708.35031 MR 1031377

23. Kloeden, P. E., \& Platen, E. Numerical Solution of Stochastic Differential Equations. Springer, Berlin (1992). Zbl 0752.60043 MR 1214374

24. Kohn, R., \& SERfATY, S. A deterministic-control-based approach to motion by curvature. Comm. Pure Appl. Math. 59 (2006), 344-407. Zbl pre05023699 MR 2200259

25. Merriman, B., Bence, J., \& Osher, S. Diffusion generated motion by mean curvature. In: The Computational Crystal Growers Workshop, Amer. Math. Soc., Providence (1992), 73-83. MR 1224451

26. Nochetto, R. H., \& VERDI, C. Convergence past singularities for a fully discrete approximation of curvature driven interfaces. SIAM J. Numer. Anal. 34 (1997), 490-512. Zbl 0876.35053 MR 1442924

27. Osher, S., \& Fedkiw, R. P. Level Set Methods and Dynamic Implicit Surfaces. Springer, New York (2003). Zbl 1026.76001 MR 1939127

28. OSher, S., \& SETHIAN, J. A. Fronts propagating with curvature-dependent speed: algorithms based on Hamilton-Jacobi formulations. J. Comput. Phys. 79 (1988), 12-49. Zbl 0659.65132 MR 0965860

29. Sethian, J. A. Level Set Methods and Fast Marching Methods: evolving interfaces in geometry, fluid mechanics, computer vision, and materials science. Cambridge Univ. Press, Cambridge (1999). Zbl 0973.76003 MR 1700751

30. Soner, H. M., \& TouzI, N. A stochastic representation for mean curvature type geometric flows. Comm. Partial Differential Equations 27 (2002), 2031-2053. Zbl 1036.49010 MR 1941665 
31. Soner, H. M., \& TouzI, N. Dynamic programming for stochastic target problems and geometric flows. J. Eur. Math. Soc. 4 (2002), 201-236. Zbl 1003.49003 MR 1924400

32. Souganidis, P. E. Front propagation: theory and applications. In [5], pp. 186-242. Zbl 0882.35016 MR 1462703

33. StAniforth, A. N., \& Côté, J. Semi-Lagrangian integration schemes for atmospheric modelsA review. Monthly Weather Rev. 119 (1991), 2206-2223.

34. Strain, J. Semi-Lagrangian methods for level set equations. J. Comput. Phys. 151 (1999), 498-533. Zbl 0942.76060 MR 1686375 